\title{
The Impact of Quasi-Equally Spaced Sensor Topologies on Signal Reconstruction
}

\author{
ALESSANDRO NORDIO and CARLA-FABIANA CHIASSERINI \\ Politecnico di Torino \\ and \\ EMANUELE VITERBO \\ Università della Calabria
}

\begin{abstract}
A wireless sensor network with randomly deployed nodes can be used to provide an irregular sampling of a physical field of interest. We assume that a sink node collects the data gathered by the sensors and uses a linear filter for the reconstruction of a bandlimited scalar field defined over a $d$-dimensional domain. Sensors' locations are assumed to be known at the sink node, up to a certain position error. We then take the mean square error (MSE) of the reconstructed field as performance metric, and evaluate the effect of both uniform and quasi-equally spaced sensor layouts on the quality of the reconstructed field. We define a parameter that provides a measure of the regularity of the sensors deployment, and, through asymptotic analysis, we derive the MSE in the case of different sensor spatial distributions. For two of them, an approximate closed form expression is obtained. We validate our analysis through numerical results, and we show that an excellent match exists between analysis and simulation even for a small number of sensors.
\end{abstract}

Categories and Subject Descriptors: C.4 [Performance of Systems]: Performance attributes; G.1.0 [Numerical Analysis]: General; G.1.2 [Numerical Analysis]: Approximation

General Terms: Design, Performance, Theory

Additional Key Words and Phrases: Sensor networks, irregular sampling, signal reconstruction, performance evaluation

\section{ACM Reference Format:}

Nordio, A., Chiasserini, C.-F., and Viterbo, E. 2010. The impact of quasi-equally spaced sensor topologies on signal reconstruction. ACM Trans. Sensor Netw. 6, 2, Article 11 (February 2010), 31 pages. DOI $=10.1145 / 1689239.1689241$ http://doi.acm.org/10.1145/1689239.1689241

This article is an extended version of the work presented at the Poster Session of IPSN 2007. This work was supported partially by the Regione Piemonte (Italy) through the VICSUM project and partially by the European Commission in the framework of the FP7 Network of Excellence in Wireless COMmunications NEWCOM++ (contract n. 216715).

Authors' addresses: A. Nordio and C.-F. Chiasserini, Dipartimento di Elettronica, Politecnico di Torino, C. Duca degli Abruzzi 24, 10129 Torino, Italy, email: \{alessandro.nordio, carla.chiasserini\}@polito.it; E. Viterbo, DEIS-Università della Calabria, Via P. Bucci, Cubo 42C, 87036 Rende (CS), Italy; email: viterbo@deis.unical.it.

Permission to make digital or hard copies of part or all of this work for personal or classroom use is granted without fee provided that copies are not made or distributed for profit or commercial advantage and that copies show this notice on the first page or initial screen of a display along with the full citation. Copyrights for components of this work owned by others than ACM must be honored. Abstracting with credit is permitted. To copy otherwise, to republish, to post on servers, to redistribute to lists, or to use any component of this work in other works requires prior specific permission and/or a fee. Permissions may be requested from Publications Dept., ACM, Inc., 2 Penn Plaza, Suite 701, New York, NY 10121-0701 USA, fax +1 (212) 869-0481, or permissions@acm.org. (C) 2010 ACM 1550-4859/2010/02-ART11 $\$ 10.00$

DOI 10.1145/1689239.1689241 http://doi.acm.org/10.1145/1689239.1689241 


\section{INTRODUCTION}

Irregular sampling theory is concerned with the problem of recovering a bandlimited signal from a sequence of samples, which may be taken in an irregular way. In this context, several reconstruction algorithms have been proposed in the literature (see e.g., Feichtinger et al. [1995]) and have found application in a variety of fields, such as digital medical imaging [Strohmer 1993; Strohmer et al. 1996], geophysics [Rauth and Strohmer 1997], weather forecast [Daley 1991], astronomy, and oceanography [Akyildiz et al. 2002].

Irregular sampling finds application also in sensor networks, where nodes sample a physical field, like air temperature, light intensity, pollution levels or rain falls. Indeed, in general, sensors are not regularly deployed in the area of interest and, thus, the physical field is not regularly sampled. Sensors report the data to a common processing unit (or sink node), which is in charge of reconstructing the sensed field. If the field is bandlimited, then an estimate of the discrete spectrum can be provided, although high sampling irregularities typically result in a degradation of the reconstructed signal. The work in Ganesan et al. [2004] investigates this issue showing how irregular spatiotemporal sampling affects the performance of sensor networks. Other interesting studies can be found in Zhao et al. [2006] and Early and Long [2001], just to name a few, which address the perturbations of regular sampling in shift-invariant spaces [Zhao et al. 2006] and the reconstruction of irregularly sampled images in presence of measure noise [Early and Long 2001].

In this work, we focus on wireless sensor networks and investigate a reconstruction technique that follows the work by Feichtinger et al. [1995]. We assume that sensors are randomly deployed on a $d$-dimensional domain (in the practice, $d=1, \ldots, 4$ ), and that their locations are known at the sink node up to a certain position error. Note that this is often the case when the sensors positions are estimated through a localization technique [Moore et al. 2004; Liu et al. 2006]. We highlight that the random deployment of the sensors and the imperfect knowledge of their positions at the sink are totally separate issues; indeed, the latter only depends on the accuracy of the localization estimation technique. We consider that the field samples are corrupted by additive noise, due to quantization, round-off errors or poor quality of the sensing devices. Furthermore, we assume that samples are transferred to the sink without suffering losses, that is, we do not consider the effects of the network dynamics, such as packet loss, network congestion, or time varying channels, on the message transfer.

Our goal is to evaluate the performance of the proposed reconstruction technique in the presence of quasi-equally spaced sensor layouts, that is, when the random distribution of the sensors over the $d$-dimensional domain is such that the sensors locations are very close to those obtained with an equally spaced deployment in each dimension. The motivation for considering such network topologies is that, typically, terrain conditions and deployment practicality make regular sensors placement unfeasible. For the sake of comparison, we also study the case of sensors randomly distributed in the network area with a uniform distribution. 
Our main contributions are as follows.

-We model the sampling system represented by the sensor network, when randomly deployed nodes sense a scalar field defined over a $d$-dimensional domain.

- Through asymptotic analysis, we derive the mean square error (MSE) on the reconstructed signal-a parameter commonly used to measure the accuracy of estimates. The MSE is obtained as a function of the network characteristics so as to evaluate the impact of different (quasi-equally spaced) sensor layouts on the quality of the field estimate.

-We introduce the parameter $\tau$, which provides a measure of the regularity of the sensor layout (i.e., of the field sampling) and we show the analytic derivation of $\tau$ for the network topologies under study.

-We find an approximate, closed form expression of the MSE for the sensor distributions under study, which proves to be very tight.

We highlight that our results play a key role in the design of sensor networks and that very few works have analytically studied the impact of random sensor layouts on the field reconstruction. This article and our previous work [Nordio et al. 2008] have a common background: both consider the reconstruction of a physical field sampled by a sensor network whose nodes, or sensors, are randomly deployed. In particular, in Nordio et al. [2008], we studied the performance of reconstruction techniques, in terms of MSE when various types of linear filters are employed to reconstruct the field from the irregularly spaced noisy samples provided by the network. In Nordio et al. [2008], sensors were considered to be randomly deployed with uniform distribution in the 1-dimensional domain $[-1 / 2,1 / 2)$ and subject to unpredictable motion.

Here, instead, we evaluate the performance of one reconstruction technique based on linear filtering (i.e., the LMMSE filter providing the best performance, according to Nordio et al. [2008]) in the scenario where sensors are stationary and quasi-equally spaced over a $d$-dimensional domain. We also highlight that making different assumptions on the sensors deployment leads to a new mathematical derivation of the system performance with respect to Nordio et al. [2008].

The rest of the article is organized as follows. Section 2 reviews some related work. Section 3 introduces the system model and our performance metric; it also recalls some details on the field reconstruction technique. In Section 4, we introduce and describe the network topology distributions under study. Section 5 presents the asymptotic analysis of the system performance, and validates the analytical results through numerical simulations. Finally, Section 6 concludes the article.

\section{RELATED WORK}

Relevant to our work is the literature on spectral analysis where, however, most of the works deal with regularly sampled signals (see e.g., Stoica and Moses [2000] and references therein). An excellent guide to irregular sampling is Marvasti [2001], which covers a large number of techniques, algorithm, and 
applications. Reconstruction techniques for irregularly sampled signals include linear [Feichtinger et al. 1995] and non-linear [Rauhut 2007] methods, iterative algorithms [Feichtinger and Gröchenig 1993], and interpolation, just to name a few. In particular, in Feichtinger and Gröchenig [1993] provide an error analysis of an iterative reconstruction algorithm taking into account round-off errors, jitters, truncation errors and aliasing. From the theoretical point of view, some theoretical works on random sampling of trigonometric polynomials can be found in Rauhut [2007], while, in the field of learning theory, an interesting work is presented in Smale and Zhou [2004]. Nevertheless, the reconstruction of non-equally spaced sampled signals is still far from being fully understood.

In the context of sensor networks, the field reconstruction at the sink node with spatial and temporal correlation among sensor measures is studied in Cristescu and Vetterli [2005], Sung et al. [2005], Vuran et al. [2004], and Rachlin et al. [2005]. In particular, in Rachlin et al. [2005] the observed field is a discrete vector of target positions and sensor observations are dependent. By modeling the sensor network as a channel encoder and exploiting some concepts from coding theory, the network capacity, defined as the maximum ratio of target positions to number of sensors, is studied as a function of noise, sensing function and sensor connections. The paper by Dong et al. [2006] considers a dense sensor network where a MAC protocol is responsible to collect samples from network nodes. The work analyzes the impact of deterministic and random data collection strategies on the quality of field reconstruction. As a performance measure, the maximum of the reconstruction square error over the sensed field is employed, as opposed to our work where the mean square error is considered. Also, in Dong et al. [2006], the field is a Gaussian random process, the exact sensor locations are known and the sink always receives a sufficiently large number of samples.

The problem of reconstructing a band-limited signal from an irregular set of samples at unknown locations is addressed in Marziliano and Vetterli [2000]. There, the field is sampled with unknown irregularly spaced sample locations. Several types of irregular sample sets are considered, always with locations randomly chosen over a discrete equally-spaced domain. Different methods for reconstructing the signal and estimating the sample locations are proposed, and the conditions for which there exist multiple solutions or a unique solution are discussed. The study in Marziliano and Vetterli [2000] also considers the case where sample locations are affected by jitter. Note that, differently from Marziliano and Vetterli [2000], we consider that the field varies over a finite $d$-dimensional domain and that the field measurements are affected by noise. Most importantly, we do not restrict the sample locations to a discrete domain.

The work in Dogandži'c and Zhang [2005] presents a Bayesian framework that accounts for sensor localization errors. The algorithms they propose are robust to these errors and allow to estimate the parameters of a partly linear signal over a bidimensional area where nodes are uniformly distributed. In Taylor et al. [2006], instead, a Bayesian filter is used to provide probabilistic on-line estimate of both sensor locations and target tracking. Finally, we point out that the problem of uncertain sensor locations is related to the problem 
of jittered sampling in the spectral analysis literature, which has been investigated in both the cases of regular and irregular sampling (see e.g., Liu and Stanley [1965]).

To summarize, the main differences between our work and the results that are available in the spectral analysis literature are the following:

(i) the analysis of the asymptotic MSE on a signal that varies over a continuous $d$-dimensional domain and is reconstructed from randomly (but quasi-equally spaced) samples,

(ii) the application of results from the theory of random matrices to the field reconstruction problem,

(iii) the focus on a Bayesian model for the (random) sensor locations, as opposed to the frequentist model of deterministic unknown locations that one encounters more commonly in the spectral analysis literature.

Finally, we would like to highlight the differences between this article and the work in Nordio et al. [2008]. In Nordio et al. [2008], we studied the reconstruction of a physical field over a unidimensional domain, sampled by a sensor network with uniformly distributed nodes and using different linear filters. Note that, although this article and Nordio et al. [2008] share a common background and employ similar mathematical tools, here we focus on the LMMSE filter, which was shown in Nordio et al. [2008] to provide the best performance, and consider different spatial sensor distributions, that is, quasi-equally spaced deployments over a $d$-dimensional domain. The different assumptions we make in this work lead to novel mathematical derivations and results. More specifically, with respect to Nordio et al. [2008], we derive a new expression for the reconstruction MSE (due to the quasi-equally spaced deployments), a measure of the sensor deployment regularity and its impact on the MSE performance, and a tight closed form approximation for the MSE.

\section{PRELIMINARIES}

Below, we first present our notations, the system model and the assumptions we make. Then, we introduce the performance metric and the reconstruction technique adopted in this work.

\subsection{Notations}

Column vectors are denoted by bold lowercase letters, while matrices are denoted by bold upper case letters. The $\left(\ell, \ell^{\prime}\right)$ entry of the generic matrix $\mathbf{Z}$ is denoted by $(\mathbf{Z})_{\ell, \ell^{\prime}}$. The $n \times n$ identity matrix is denoted by $\mathbf{I}_{n} \cdot(\cdot)^{\mathrm{T}}$ is the transpose operator, while (.) $)^{\dagger}$ is the conjugate transpose operator. We denote by $f_{x}(z)$ the probability density function (pdf) of the generic random variable $x$, and by $\mathbb{E}[\cdot]$ the average operator.

\subsection{System Model}

Let us consider a field defined over a $d$-dimensional domain. A physical field is approximately bandlimited and, when observed over a finite interval, it admits 
an infinite Fourier series expansion. However, one can truncate the Fourier series so as to consider only the non-negligible coefficients. Let us denote by $M$ the largest index in the truncated sum; this represents the approximate one-sided bandwidth of the field.

We therefore represent the field, $s(\mathbf{x})$, by using $2 M+1$ harmonics per dimension as

$$
s(\mathbf{x})=\frac{1}{\sqrt{(2 M+1)^{d}}} \sum_{\ell} a_{\nu(\ell)} \mathrm{e}^{\mathrm{j} 2 \pi \ell^{\mathrm{T}} \mathbf{x}}
$$

where $\ell=\left[\ell_{1}, \ldots, \ell_{d}\right]^{\mathrm{T}}$ is a vector of integers with $\ell_{m}=-M, \ldots, M$. The term $\sum_{\ell}$ represents a $d$-dimensional sum, and the function

$$
v(\ell)=\sum_{m=1}^{d}(2 M+1)^{m-1} \ell_{m}
$$

maps the vector $\ell$ onto a scalar index. Notice that $-N \leq v(\ell) \leq+N$, and that

$$
N=\frac{(2 M+1)^{d}-1}{2}
$$

The field is observed within the hypercube $\mathcal{H}=\left\{\mathbf{x} \mid \mathbf{x} \in[-1 / 2,1 / 2)^{d}\right\}$ and $r$ samples taken by sensors placed at positions $\left\{\mathbf{x}_{1}, \ldots, \mathbf{x}_{r}\right\}$, with $\mathbf{x}_{q}=$ $\left[x_{1, q}, \ldots, x_{d, q}\right]^{\mathrm{T}} \in \mathcal{H}$ and $q=1, \ldots, r$, are available at the sink node. Notice that this assumption reflects the case where a set of sensors send to the sink node a data message every "report period" and, at each report period, the sink reconstructs the signal from the received samples. Given a report period, we therefore assume that $r$ measures are delivered to the sink, and we do not consider the effects of the network dynamics, such as packet loss, network congestion, or time varying channels, on the message transfer.

Let $\mathbf{s}=\left[s_{1}, \ldots, s_{r}\right]^{\mathrm{T}}, s_{q}=s\left(\mathbf{x}_{q}\right)$, the values of the $r$ field samples received at the sink. The field discrete spectrum is denoted by the random vector $\mathbf{a}=$ $\left[a_{-N}, \ldots, a_{+N}\right]^{\mathrm{T}}$, and we assume $\mathbb{E}\left[\mathbf{a a}^{\dagger}\right]=\sigma_{a}^{2} \mathbf{I}_{(2 M+1)^{d}}$. Furthermore, we define $\beta$ as the ratio of the double-sided bandwidth of the field to the number of sensors:

$$
\beta=\frac{(2 M+1)^{d}}{r}
$$

Notice that for equally spaced sensors, the sampling theorem is satisfied for $\beta \leq 1$. We therefore limit our analysis to the range $0<\beta \leq 1$.

We collect the sampling positions in the columns of the $d \times r$ matrix $\mathbf{X}$, given by $\mathbf{X}=\left[\mathbf{x}_{1}, \ldots, \mathbf{x}_{r}\right]$. Following [Feichtinger et al. 1995], the values of the field at positions $\mathbf{x}_{1}, \ldots, \mathbf{x}_{r}$ are given by $\mathbf{s}=\mathbf{G}_{\mathbf{X}}^{\dagger} \mathbf{a}$ where $\mathbf{G}_{\mathbf{X}}$ is the $(2 M+1)^{d} \times r$ matrix defined as:

$$
\left(\mathbf{G}_{\mathbf{X}}\right)_{v(\ell), q}=\frac{1}{\sqrt{(2 M+1)^{d}}} \mathrm{e}^{-\mathrm{j} 2 \pi \ell^{\mathrm{T}} \mathbf{x}_{q}}
$$

for $q=1, \ldots, r$. The coefficient $1 / \sqrt{(2 M+1)^{d}}$ is a normalization factor. As indicated by the subscript, the matrix $\mathbf{G}_{\mathbf{X}}$ is a function of the matrix $\mathbf{X}$. 
When the measures $\mathbf{p}$, provided by the sensors, are affected by noise, we can write:

$$
\mathbf{p}=\mathbf{s}+\mathbf{n}=\mathbf{G}_{\mathbf{X}}^{\dagger} \mathbf{a}+\mathbf{n}
$$

where the noise is represented by the size $r$, zero-mean random vector $\mathbf{n}$, with covariance matrix $\mathbb{E}\left[\mathbf{n n}^{\dagger}\right]=\sigma_{n}^{2} \mathbf{I}_{r}$.

We define the signal-to-noise ratio on the measure as:

$$
\mathrm{SNR}_{m} \triangleq \frac{\sigma_{a}^{2}}{\sigma_{n}^{2}} .
$$

For simplicity, in the following, we use the parameter $\alpha$, defined as:

$$
\alpha=\frac{1}{\mathrm{SNR}_{m}}
$$

Note that, if not available, $\mathrm{SNR}_{m}$ can be obtained through the unbiased estimator proposed in Kobayashi et al. [2001, eq. (6)] as $\mathrm{SNR}_{m}=$ $\frac{1}{r} \sum_{q=1}^{r}\left|p_{q}\right|^{2} / \sigma_{n}^{2}-1$, where $\sigma_{n}^{2}$ is estimated based on the sensor node in use. In Section 5.1, we show that the proposed system is very robust to mismatches in the $\mathrm{SNR}_{m}$ estimation and that the degradation in the performance due to such mismatch is negligible.

Measures $\mathbf{p}$ are sent to the sink node, whose task is to provide an accurate field reconstruction, that is, an estimate of the field value for any generic location in hypercube $\mathcal{H}$. Data transmissions between sensors and sink node are considered to be error free. We assume that the sink does not have perfect knowledge of the network topology and that an estimate of the sensor positions is given by

$$
\hat{\mathbf{X}}=\mathbf{X}+\Delta,
$$

where the position error is denoted by the $d \times r$ matrix $\Delta=\left[\delta_{1}, \ldots, \delta_{r}\right]$. The entries of the matrix $\Delta, \delta_{m, q}$, are modeled as independent and identically distributed, zero-mean random variables with variance $\sigma_{\delta}^{2}$ and $\mathrm{pdf} f_{\delta}(z)$, such that $f_{\delta}(z)=f_{\delta}(-z)$. The columns of the matrix $\Delta$ and the random vectors $\mathbf{n}$ and $\mathbf{a}$ are assumed to be mutually independent. Note that our problem differs from the well known problem of jittered sampling (see e.g., Liu and Stanley [1965]), since we deal with irregular sample locations.

\subsection{Performance Metric and Reconstruction Techniques}

The task of the reconstruction algorithm is to compute an estimate $\hat{s}(\mathbf{x})$ of the field $s(\mathbf{x})$, or, equivalently, an estimate $\hat{\mathbf{a}}$ of the spectrum a. Given $\hat{\mathbf{a}}$, the field estimates $\hat{s}(\mathbf{x})$ for any location $\mathbf{x}$ can be obtained as

$$
\hat{s}(\mathbf{x})=\frac{1}{\sqrt{(2 M+1)^{d}}} \sum_{\ell} \hat{a}_{v(\ell)} \mathrm{e}^{\mathrm{j} 2 \pi \ell^{\mathrm{T}} \mathbf{x}} .
$$

Thus, a high-quality field reconstruction corresponds to an accurate estimate of a. A parameter commonly used to measure the accuracy of estimates is the 
mean square error. In our case, the MSE on the field spectrum estimate is defined as:

$$
\mathrm{MSE}_{\mathbf{X}}=\int_{\mathcal{H}}|s(\mathbf{x})-\hat{s}(\mathbf{x})|^{2} \mathrm{~d} \mathbf{x}=\frac{\underset{\mathbf{a}, \mathbf{n}, \mathbf{\Delta}}{\mathbb{E}}\left[\|\hat{\mathbf{a}}-\mathbf{a}\|^{2}\right]}{(2 M+1)^{d}},
$$

where the operator $\mathbb{E}[\cdot]$ averages with respect to the subscripted random vectors. The MSE, as in (3), depends on the sensing positions $\mathbf{X}$ and provides a measure of the overall field reconstruction quality.

For models such as (2), various estimation techniques based on linear filtering can be used, and their performances have been widely investigated in the literature (see e.g., Kay [1993]). In general, the idea is to employ a suitable filter B such that the estimate of the field spectrum is given by the linear operation

$$
\hat{\mathbf{a}}=\mathbf{B}^{\dagger} \mathbf{p}
$$

where $\mathbf{B}$ is an $r \times(2 M+1)^{d}$ matrix.

Assuming $\mathrm{SNR}_{m}$ known at the sink, we focus on the filter providing the best performance in terms of MSE, that is, the linear minimum MSE (LMMSE) filter, ${ }^{1}$ which, in our case, is given by Kay [1993]:

$$
\mathbf{B}=\beta \mathbf{G}_{\mathbf{X}}^{\dagger}\left(\mathbf{T}_{\mathbf{X}}+\alpha \beta \mathbf{I}\right)^{-1}
$$

where the $(2 M+1)^{d} \times(2 M+1)^{d}$ matrix $\mathbf{T}_{\mathbf{X}}=\beta \mathbf{G}_{\mathbf{X}} \mathbf{G}_{\mathbf{X}}^{\dagger}$ is Block Toeplitz and Hermitian, with

$$
\left(\mathbf{T}_{\mathbf{X}}\right)_{v(\ell), v\left(\ell^{\prime}\right)}=\frac{1}{r} \sum_{q=1}^{r} \mathrm{e}^{-\mathrm{j} 2 \pi\left(\ell-\ell^{\prime}\right)^{\mathrm{T}} \mathbf{x}_{q}}
$$

Notice that (5) requires the knowledge of $\mathbf{X}$, which is not available at the sink. We then replace it by its estimate $\hat{\mathbf{X}}$, obtaining a suboptimal signal reconstruction. The employed filter is given by:

$$
\mathbf{B}=\beta \mathbf{G}_{\hat{\mathbf{x}}}^{\dagger}\left(\mathbf{T}_{\hat{\mathbf{x}}}+\alpha \beta \mathbf{I}\right)^{-1}
$$

Here, the matrix $\mathbf{G}_{\hat{\mathbf{x}}}$ is defined as in (1), where $\mathbf{x}_{q}$ is replaced by $\hat{\mathbf{x}}_{q}$, and $\mathbf{T}_{\hat{\mathbf{x}}}=\beta \mathbf{G}_{\hat{\mathbf{x}}} \mathbf{G}_{\hat{\mathbf{x}}}^{\dagger}$. Notice that the reconstruction technique we adopt is conditionally linear, that is, it is linear once the position estimates are given.

From now on, for the sake of notation simplicity, we remove the subscripts $\mathbf{X}$ and $\hat{\mathbf{X}}$ and we define $\mathbf{G} \triangleq \mathbf{G}_{\mathbf{X}}, \widehat{\mathbf{G}} \triangleq \mathbf{G}_{\hat{\mathbf{X}}}, \mathbf{T} \triangleq \mathbf{T}_{\mathbf{X}}, \widehat{\mathbf{T}} \triangleq \mathbf{T}_{\hat{\mathbf{X}}}$.

\footnotetext{
${ }^{1}$ Although the following analysis is based on the LMMSE filter, it can be easily generalized to any linear filter.
}

ACM Transactions on Sensor Networks, Vol. 6, No. 2, Article 11, Publication date: February 2010. 
Substituting (2) and (4) in (3), after some algebra, the MSE can be written as:

$$
\begin{aligned}
\operatorname{MSE}_{\mathbf{X}}= & \frac{\underset{\mathbf{a}, \mathbf{n}, \Delta}{\mathbb{E}}\left[\left\|\mathbf{B}^{\dagger}\left(\mathbf{G}^{\dagger} \mathbf{a}+\mathbf{n}\right)-\mathbf{a}\right\|^{2}\right]}{(2 M+1)^{d}} \\
= & \frac{\sigma_{a}^{2}}{(2 M+1)^{d}} \operatorname{Tr}\left\{\beta^{2} \underset{\Delta}{\mathbb{E}}\left[\mathbf{G} \widehat{\mathbf{G}}^{\dagger}(\widehat{\mathbf{T}}+\alpha \beta \mathbf{I})^{-2} \widehat{\mathbf{G}} \mathbf{G}^{\dagger}\right]+\alpha \beta \underset{\Delta}{\mathbb{E}}\left[\widehat{\mathbf{T}}(\widehat{\mathbf{T}}+\alpha \beta \mathbf{I})^{-2}\right]\right. \\
& \left.+\mathbf{I}-2 \beta \Re\left\{\underset{\Delta}{\mathbb{E}}\left[\mathbf{G} \widehat{\mathbf{G}}^{\dagger}(\widehat{\mathbf{T}}+\alpha \beta \mathbf{I})^{-1}\right]\right\}\right\},
\end{aligned}
$$

where $\operatorname{Tr}\{\cdot\}$ and $\Re\{\cdot\}$ are the trace and real-part operators, respectively. The above expression for the MSE will be used to derive our asymptotic analysis.

The main parameters introduced in this section are summarized in Appendix A.

\section{RANDOM TOPOLOGY DISTRIBUTION}

Equation (7) clearly is a function of a deterministic network topology defined by $\mathbf{X}$. From now on, we treat the matrix $\mathbf{X}$ as random, that is, we assume the topology to be random, with independent positions $x_{m, q}=(\mathbf{X})_{m, q}$ following the pdf $f_{x_{m, q}}(z), m=1, \ldots, d, q=1, \ldots, r$. Also, let us denote by $\overline{\mathbf{X}}=\mathbb{E}[\mathbf{X}]$ the average value of $\mathbf{X}$.

In particular, we assume that the sensors, from which the field samples are received at the sink node, are quasi-equally spaced on a $d$-dimensional domain, that is, they have jittered locations around the vertices of a $d$-dimensional regular grid topology. ${ }^{2}$ A more formal definition can be given as follows. Let us denote by $r^{\prime}=r^{\frac{1}{d}}$ the number of samples per spatial dimension. We characterize the class of random topologies under study through the following properties:

$-\mathbf{X}-\overline{\mathbf{X}}=\frac{1}{r^{\prime}} \tilde{\mathbf{X}}$, where $(\tilde{\mathbf{X}})_{m, q}=\tilde{x}_{m, q}(m=1, \ldots, d, q=1, \ldots, r)$ are random variables;

— each column $\overline{\mathbf{x}}_{q}$ of $\overline{\mathbf{X}}(q=1, \ldots, r)$ corresponds to a vertex of a $d$-dimensional regular grid in in $\mathcal{H}$;

- the random variables $\tilde{x}_{m, q}$ are distributed with pdf $f_{\tilde{x}_{m, q}}(z)$, which has zero mean and does not depend on $m$ or $q$.

Note that, by defining the vector $\mathbf{q}^{\prime}=\left[q_{1}^{\prime}, \ldots, q_{d}^{\prime}\right]$, with $q_{m}^{\prime}=1, \ldots, r^{\prime}$, and the mapping function: $\mu\left(\mathbf{q}^{\prime}\right)=\sum_{m=1}^{d}\left(r^{\prime}\right)^{m-1} q_{m}^{\prime}$, we can write the mean value of $x_{m, q}$ as:

$$
\bar{x}_{m, q}=\bar{x}_{m, \mu\left(\mathbf{q}^{\prime}\right)}=\frac{q_{m}^{\prime}-1 / 2}{r^{\prime}}-\frac{1}{2}
$$

that is, as equally spaced averages on $[-1 / 2,+1 / 2)$.

${ }^{2}$ Recall that here we do not address issues related to the data transfer from the sensors to the sink node. 


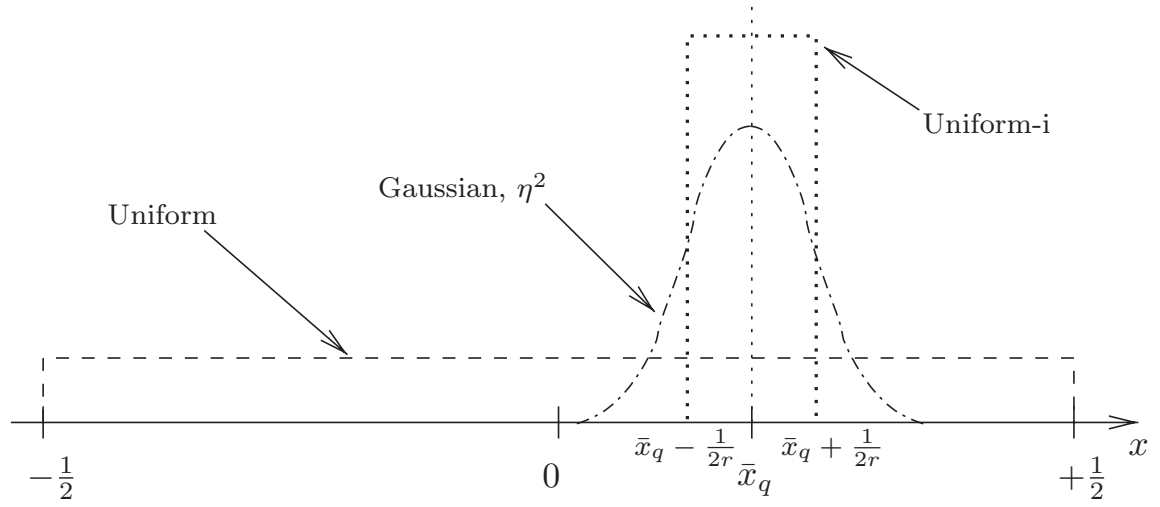

Fig. 1. Qualitative representations of the distributions Uniform, Uniform-i and Gaussian in a unidimensional scenario $(d=1)$.

As case study, we consider the following topology distributions:

-Uniform-i: Where $x_{m, q}=\bar{x}_{m, q}+\tilde{x}_{m, q} / r^{\prime}$ is uniformly distributed in the interval $\left[\bar{x}_{m, q}-1 / 2 r^{\prime}, \bar{x}_{m, q}+1 / 2 r^{\prime}\right)$. The variable $\tilde{x}_{m, q}$ is uniformly distributed in $[-1 / 2,1 / 2)$ and has variance $1 / 12$.

-Gaussian: Where $x_{m, q}=\bar{x}_{m, q}+\tilde{x}_{m, q} / r^{\prime}$ and $\tilde{x}_{m, q}$ is Gaussian distributed with zero mean and variance $\eta^{2}$.

For the sake of comparison, we also consider the Uniform case, which is widely used in the literature and assumes that each sensor is deployed with uniform distribution over the entire sampling region, that is,

-Uniform: Where $\mathbb{E}[\mathbf{X}]=\mathbf{0}$ and $x_{m, q}$ has zero mean and is uniformly distributed in $[-1 / 2,1 / 2)$.

A graphical representation of the above distributions is presented in Figure 1, for $d=1$. The figure shows the distributions of the $q$-th sensor position, for the three mentioned topologies. The mean of the Gaussian and Uniform-i distributions is given by $\bar{x}_{q}$, while the Uniform distribution has zero mean and support in $[-1 / 2,1 / 2)$. The Uniform-i distribution has support in $\left[\bar{x}_{q}-1 / 2 r, \bar{x}_{q}-1 / 2 r\right)$.

Note that, one of the reasons for analyzing the Uniform distribution, is that, among all distributions satisfying the above properties, it is characterized by the highest variance; thus, as will be clear in Section 5 , it represents a worst case. Instead, in our scenario, Uniform-i and Gaussian reflect the attempt to deploy sensors at equally-spaced positions $\overline{\mathbf{X}}$, with $\tilde{\mathbf{X}} / r^{\prime}$ representing the deployment error, while $\tilde{\mathbf{X}}$ is the deployment error normalized to the average sensor separation (per dimension), $1 / r^{\prime}$. Notice that the support of the Gaussian pdf is infinite and, thus, with non-zero probability some of the samples $\mathbf{x}$ fall outside the region $\mathcal{H}$. In principle, such values should be discarded. In practice, in our work, they are considered to be valid sampling points by wrapping them around the region $[-1 / 2,1 / 2)^{d}$. Note that, when the variance $\eta^{2}$ is small, the effect of such operation is negligible.

ACM Transactions on Sensor Networks, Vol. 6, No. 2, Article 11, Publication date: February 2010. 
In the following, the three distributions are compared in terms of the MSE obtained on the reconstructed field. Clearly, both the noise $\mathbf{n}$ and the position error $\Delta$ affect the quality of the reconstructed field. High reconstruction quality is expected when the standard deviation $\sigma_{\delta}$ is small compared to the average sample separation. We therefore introduce the parameter

$$
\rho=\frac{\sigma_{\delta}}{1 / r^{\prime}}
$$

as a measure of the sampling uncertainty on the network topology at the sink. When $\rho=0$ (i.e., $\sigma_{\delta}=0$ ), we have perfect knowledge on the network topology.

Notice that the error $\Delta$ in the knowledge of the node positions appears when node localization techniques are employed to estimate the sensors positions. As an example, an iterative localization technique is proposed in Liu et al. [2006], where network topologies very similar to those studied in this article are considered and some values for the error variance $\sigma_{\delta}$ are provided.

\section{ASYMPTOTIC ANALYSIS OF THE MSE OF THE PROPOSED RECONSTRUCTION TECHNIQUE}

A simple and effective tool for evaluating the performance of large finite systems is asymptotic analysis [de Bruijn 1958]. In our case, we let the number of field harmonics and the number $r$ of sensors grow to infinity, while the ratio $\beta=$ $(2 M+1)^{d} / r$ is kept constant. The numerical results provided in this section show the validity of our asymptotic analysis, even for small values of $M$ and $r$.

As performance metric, we consider the asymptotic MSE, defined as:

$$
\mathrm{MSE}_{\infty}=\lim _{M, r \rightarrow+\infty} \frac{\underset{\beta}{\mathbb{\mathbf { x }}}\left[\mathrm{MSE}_{\mathbf{X}}\right]}{\sigma_{a}^{2}}
$$

Also, for simplicity of notation, we introduce the linear functional [Tulino and Verdú 2004]

$$
\phi(\mathbf{Y})=\lim _{n \rightarrow+\infty} \frac{1}{n} \operatorname{Tr}\{\mathbb{E}[\mathbf{Y}]\},
$$

where the argument $\mathbf{Y}$ is an $n \times n$ random square matrix and the operator $\mathbb{E}[\cdot]$ averages over the random variables in $\mathbf{Y}$. The functional $\phi(\cdot)$ has some interesting properties; here, we report only those we use in our analysis:

$$
\phi(\mathbf{I})=1, \quad \phi(g(\mathbf{Y}))=\mathbb{E}\left[g\left(y_{i}\right)\right] ; \quad \phi\left(a_{1} \mathbf{Y}_{1}+a_{2} \mathbf{Y}_{2}\right)=a_{1} \phi\left(\mathbf{Y}_{1}\right)+a_{2} \phi\left(\mathbf{Y}_{2}\right),
$$

where $y_{i}$ is the random variable distributed as the asymptotic eigenvalues of $\mathbf{Y}$, $g(y)$ is an analytic function, defined in $y>0$, and $a_{1}, a_{2}$ are scalars (see Tulino and Verdú [2004] for further details). From (7) and using the above definitions, we obtain:

$$
\begin{aligned}
\operatorname{MSE}_{\infty}=\beta^{2} \phi & \left(\mathbf{G} \widehat{\mathbf{G}}^{\dagger}(\widehat{\mathbf{T}}+\alpha \beta \mathbf{I})^{-2} \widehat{\mathbf{G}} \mathbf{G}^{\dagger}\right)+\alpha \beta \phi\left(\widehat{\mathbf{T}}(\widehat{\mathbf{T}}+\alpha \beta \mathbf{I})^{-2}\right) \\
& +1-2 \beta \Re \phi\left(\mathbf{G} \widehat{\mathbf{G}}^{\dagger}(\widehat{\mathbf{T}}+\alpha \beta \mathbf{I})^{-1}\right) .
\end{aligned}
$$

Looking at (10), we observe that the asymptotic MSE depends on the ratio $\beta$ of the number of field harmonics to the number of samples, on the sensor 
measures quality $\left(\mathrm{SNR}_{m}\right)$ and on the sensor locations (through the matrices $\mathbf{G}$ and $\mathbf{T}$ ). Next, we analyze the cases where $x$ follows the Uniform, Uniform-i and Gaussian distributions.

\subsection{The Uniform Topology Distribution}

Here, we derive the expression of the asymptotic MSE when sensors are uniformly distributed on the $d$-dimensional domain. We start from (10) and apply some results obtained on the matrices $\mathbf{G}$ and $\mathbf{T}$. The final expression of the $\mathrm{MSE}_{\infty}$ allows us to make some important observations on the quality of the reconstructed field, as a function of $\beta$, the $\mathrm{SNR}_{m}$ and the uncertainty on the sensor locations $(\rho)$.

Let us first present the results on $\mathbf{G}$ and $\mathbf{T}$ that hold under the assumption of Uniform distribution:

$$
\begin{aligned}
\phi\left(\widehat{\mathbf{T}}^{p}\right) & =\phi\left(\mathbf{T}^{p}\right) \\
\phi\left(\mathbf{G} \widehat{\mathbf{G}}^{\dagger} \widehat{\mathbf{T}}^{p}\right) & =\phi\left(\widehat{\mathbf{G}}^{\dagger} \mathbf{T}^{p}\right) \\
\phi\left(\mathbf{G} \widehat{\mathbf{G}}^{\dagger} \widehat{\mathbf{T}}^{p} \widehat{\mathbf{G}}^{\dagger}\right) & =\phi\left(\widehat{\mathbf{G}}^{\dagger} \mathbf{T}^{p} \mathbf{G} \widehat{\mathbf{G}}^{\dagger}\right),
\end{aligned}
$$

where $p$ is a positive integer. The proofs are given in Appendix B. Moreover, in Appendix $\mathrm{C}$ it is shown that:

$$
\begin{aligned}
\underset{\Delta}{\mathbb{E}}[\widehat{\mathbf{G}}] & =\mathbf{C G} \\
\underset{\Delta}{\mathbb{E}}[\widehat{\mathbf{G}} \dagger & =\mathbf{G}^{\dagger} \mathbf{C}^{\dagger} \mathbf{C G}+\theta \mathbf{I},
\end{aligned}
$$

where $\theta=1-\operatorname{Tr}\left\{\mathbf{C}^{\dagger} \mathbf{C}\right\} /(2 M+1)^{d}$ and the $(2 M+1)^{d} \times(2 M+1)^{d}$ diagonal matrix $\mathbf{C}$ has entries given by: $(\mathbf{C})_{\nu(\ell), v(\ell)}=\prod_{m=1}^{d} C_{\delta}\left(-\mathrm{j} 2 \pi \ell_{m}\right)$ with $C_{\delta}(s)=$ $\int_{-\infty}^{+\infty} \exp (s z) f_{\delta}(z) \mathrm{d} z$ being the characteristic function of the random variable $\delta$.

From (11), it follows that, if the function $g(\widehat{\mathbf{T}})$ admits a power series in the variable $\widehat{\mathbf{T}}$ with expansion coefficients $c_{i}$ (i.e., $g(\widehat{\mathbf{T}})=\sum_{i=0}^{\infty} c_{i} \widehat{\mathbf{T}}^{i}$ ), then, thanks to the linearity of the functional $\phi($.$) , we have: \phi(g(\widehat{\mathbf{T}}))=\phi(g(\mathbf{T}))$. Therefore, by defining $g(\widehat{\mathbf{T}})=\widehat{\mathbf{T}}(\widehat{\mathbf{T}}+\alpha \mathbf{I})^{-2}$, the second term in (10) simplifies to $\alpha \phi\left(\mathbf{T}(\mathbf{T}+\alpha \mathbf{I})^{-2}\right)$. Similarly, for the first and fourth term in (10), we obtain: $\phi\left(\mathbf{G} \widehat{\mathbf{G}}^{\dagger} g(\widehat{\mathbf{T}}) \widehat{\mathbf{G}} \mathbf{G}^{\dagger}\right)=\phi\left(\widehat{\mathbf{G}} \mathbf{G}^{\dagger} g(\mathbf{T}) \mathbf{G} \widehat{\mathbf{G}}^{\dagger}\right)$ and $\phi\left(\mathbf{G} \widehat{\mathbf{G}}^{\dagger} g(\widehat{\mathbf{T}})\right)=\phi\left(\widehat{\mathbf{G}} \mathbf{G}^{\dagger} g(\mathbf{T})\right)$, respectively. Hence, the asymptotic MSE reduces to

$$
\begin{gathered}
\operatorname{MSE}_{\infty}=\beta^{2} \phi\left(\widehat{\mathbf{G}} \mathbf{G}^{\dagger}(\mathbf{T}+\alpha \beta \mathbf{I})^{-2} \mathbf{G} \widehat{\mathbf{G}}^{\dagger}\right)+\alpha \beta \phi\left(\mathbf{T}(\mathbf{T}+\alpha \beta \mathbf{I})^{-2}\right) \\
+1-2 \beta \Re \phi\left(\widehat{\mathbf{G}} \mathbf{G}^{\dagger}(\mathbf{T}+\alpha \beta \mathbf{I})^{-1}\right) .
\end{gathered}
$$

As shown in Appendix D, by applying to the first term in (16) the definition of $\phi(\cdot)$, the properties of the trace operator, and (15), we obtain:

$$
\begin{aligned}
\beta^{2} \phi\left(\widehat{\mathbf{G}} \mathbf{G}^{\dagger}(\mathbf{T}+\alpha \beta \mathbf{I})^{-2} \mathbf{G} \widehat{\mathbf{G}}^{\dagger}\right)= & \phi\left(\mathbf{C}^{\dagger} \mathbf{C T}(\mathbf{T}+\alpha \beta \mathbf{I})^{-2} \mathbf{T}\right) \\
& +\beta \phi\left(\theta \mathbf{T}(\mathbf{T}+\alpha \beta \mathbf{I})^{-2}\right)
\end{aligned}
$$


Similarly, using (14), the fourth term of (16) can be rewritten as:

$$
\begin{aligned}
& -2 \beta \Re \phi\left(\widehat{\mathbf{G}} \mathbf{G}^{\dagger}(\mathbf{T}+\alpha \beta \mathbf{I})^{-1}\right)=-2 \beta \Re \phi\left(\underset{\Delta}{\mathbb{E}}[\widehat{\mathbf{G}}] \mathbf{G}^{\dagger}(\mathbf{T}+\alpha \beta \mathbf{I})^{-1}\right) \\
& =-2 \beta \Re \phi\left(\mathbf{C G G} \mathbf{G}^{\dagger}(\mathbf{T}+\alpha \beta \mathbf{I})^{-1}\right) \\
& =-2 \Re \phi\left(\mathbf{C T}(\mathbf{T}+\alpha \beta \mathbf{I})^{-1}\right) .
\end{aligned}
$$

Considering all terms together, we have:

$$
\begin{gathered}
\operatorname{MSE}_{\infty}=\phi\left(\mathbf{C}^{\dagger} \mathbf{C T}(\mathbf{T}+\alpha \beta \mathbf{I})^{-2} \mathbf{T}\right)+\beta \phi\left((\theta+\alpha) \mathbf{T}(\mathbf{T}+\alpha \beta \mathbf{I})^{-2}\right) \\
+1-2 \Re \phi\left(\mathbf{C T}(\mathbf{T}+\alpha \beta \mathbf{I})^{-1}\right) .
\end{gathered}
$$

Next, let us assume that $\mathbf{C}$ and $\mathbf{T}$ are asymptotically free matrices [Tulino and Verdú 2004] (later in the paper, we will show the validity of such an assumption by comparing analytical and simulation results). Then, $\phi\left(\mathbf{C}^{p_{1}} \mathbf{T}^{p_{2}}\right)=$ $\phi\left(\mathbf{C}^{p_{1}}\right) \phi\left(\mathbf{T}^{p_{2}}\right)$ for any positive integers $p_{1}, p_{2}$. By using the properties of the functional $\phi(\cdot)$, the final expression of the asymptotic MSE becomes:

$$
\begin{aligned}
& \operatorname{MSE}_{\infty}=\phi\left(\mathbf{C}^{\dagger} \mathbf{C}\right) \phi\left(\mathbf{T}(\mathbf{T}+\alpha \beta \mathbf{I})^{-2} \mathbf{T}\right)+\beta \phi(\theta+\alpha) \phi\left(\mathbf{T}(\mathbf{T}+\alpha \beta \mathbf{I})^{-2}\right) \\
&+ 1-2 \Re\left\{\phi(\mathbf{C}) \phi\left(\mathbf{T}(\mathbf{T}+\alpha \beta \mathbf{I})^{-1}\right)\right\} \\
&=\phi\left(\mathbf{C}^{\dagger} \mathbf{C}\right) \mathbb{E}\left[\frac{\lambda^{2}}{(\lambda+\alpha \beta)^{2}}\right]+\beta\left(\alpha+1-\phi\left(\mathbf{C}^{\dagger} \mathbf{C}\right)\right) \mathbb{E}\left[\frac{\lambda}{(\lambda+\alpha \beta)^{2}}\right] \\
&+1-2 \phi(\Re\{\mathbf{C}\}) \mathbb{E}\left[\frac{\lambda}{\lambda+\alpha \beta}\right],
\end{aligned}
$$

where $\phi(\theta)=1-\phi\left(\mathbf{C}^{\dagger} \mathbf{C}\right)$ and $\lambda>0$ is a random variable with pdf $f_{\lambda}(z, \beta, d)$, distributed as the asymptotic eigenvalues ${ }^{3}$ of the matrix $\mathbf{T}$.

Notice that the relationship between $\mathbf{T}$ and $\lambda$ is expressed through the functional $\phi(\cdot)$, as in (8); thus, the pdf of $\lambda$ is strictly related to the statistical behavior of T. In (18) we applied the relation $\phi(g(\mathbf{T}))=\mathbb{E}[g(\lambda)]$ (see (9)), for $g(x)=x^{2} /(x+\alpha \beta)^{2}, g(x)=x /(x+\alpha \beta)^{2}$, and $g(x)=x /(x+\alpha \beta)$, respectively.

Moreover, looking at (18), we make the following remarks.

(i) Equation (18) shows how the distribution of the random variable $\lambda$, the noise level $\alpha$ and the imperfect knowledge on the network topology at the sink (the latter represented by the matrix $\mathbf{C}$ ) affect the MSE. The distribution of $\lambda$, which is closely related to the matrix $\mathbf{T}$ through the functional $\phi(\cdot)$, depends on the parameter $\beta$ and on the dimension $d$ of the field domain.

(ii) For the computation of the averages in (18), a closed form expression of $f_{\lambda}(z, \beta, d)$ would be required. To the best of our knowledge such expression

${ }^{3}$ Given an $n \times n$ Hermitian matrix, the empirical cumulative distribution function of its eigenvalues is defined as $F_{\lambda}^{(n)}(z)=\frac{1}{n} \sum_{i=1}^{n} 1\left\{\lambda_{i} \leq z\right\}$ where $\lambda_{1}, \ldots, \lambda_{n}$ are the eigenvalues and $1\{\cdot\}$ is the indicator function. If $F_{\lambda}^{(n)}(z)$ converges as $n \rightarrow \infty$, then the the limit is denoted by $F_{\lambda}(z)$. The corresponding asymptotic pdf is denoted by $f_{\lambda}(z)$. 


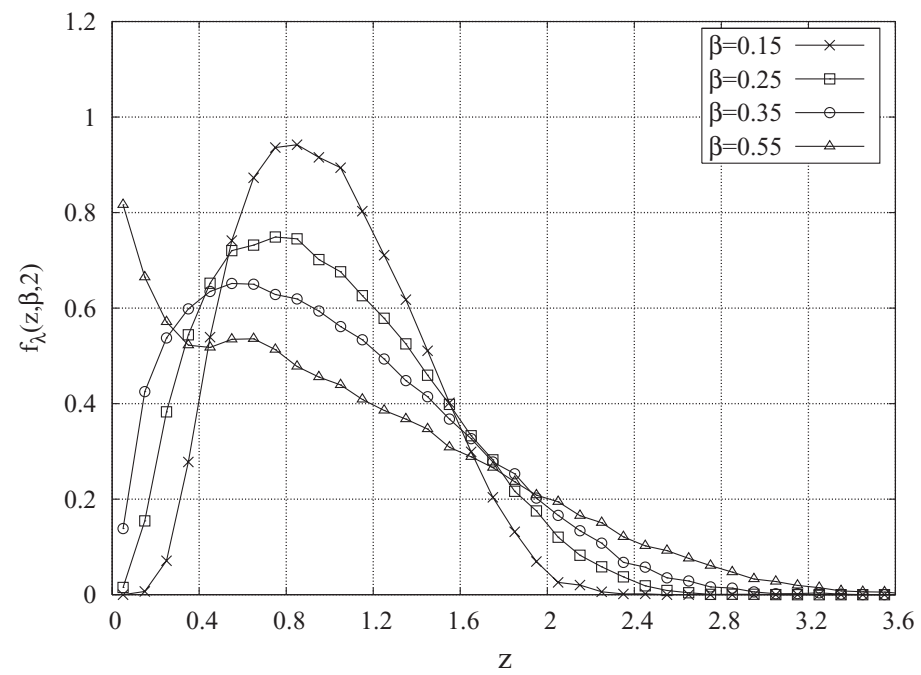

Fig. 2. Histograms of $f_{\lambda}(z, \beta, 2)$ for different values of $\beta, M=6$ and bin width $=0.1$.

is still unknown, although [Nordio et al. 2006] provides an algorithm to evaluate the moments of $\lambda$ in closed form. We compute the averages in (18) numerically.

(iii) If $\rho=0$, then (18) reduces to $\mathbb{E}[\alpha \beta /(\lambda+\alpha \beta)]$.

In the rest of this section, we present some results based on the evaluation of (18) for $d=2$. Indeed, as noted above, the asymptotic MSE in (18) is a function of the parameter $d$, in $f_{\lambda}(z, \beta, d)$. Similar results can be obtained for different values of $d$.

Figure 2 shows the histograms of $f_{\lambda}(z, \beta, d)$ for the Uniform topology distribution, $d=2$, and different values of $\beta$. Note that, as $\beta$ increases, the number of very small eigenvalues increases. This behavior is due to the fact that, with fixed $r$, the number of rows of $\mathbf{G}$ increases with $\beta$ and, by consequence, the probability of independent rows decreases. It follows that for large $\beta$ the probability of zero or close to zero eigenvalues increases [Nordio et al. 2006]. The histograms have been obtained by computing the eigenvalues of 200 realizations of the matrix $\mathbf{T}$, with $M=6$. Although $M=6$ is not a very large value, we verified that it is already sufficient to accurately estimate the asymptotic eigenvalue distribution. Observe that, for $\beta=0$ (i.e., $r \rightarrow \infty$, while keeping $M$ constant), the matrix $\mathbf{T}$ tends to the identity matrix and, thus, $f_{\lambda}(z, 0, d)=\delta_{\mathrm{D}}(z-1)$ with $\delta_{\mathrm{D}}(\cdot)$ being the Dirac's delta.

As for the matrix $\mathbf{C}$, we observe that, when the error $\delta$ is Gaussian distributed with zero mean and variance $\sigma_{\delta}^{2}$, its characteristic function, $C_{\delta}(s)$, is given by:

$$
C_{\delta}(s)=\exp \left(\sigma_{\delta}^{2} s^{2} / 2\right)
$$




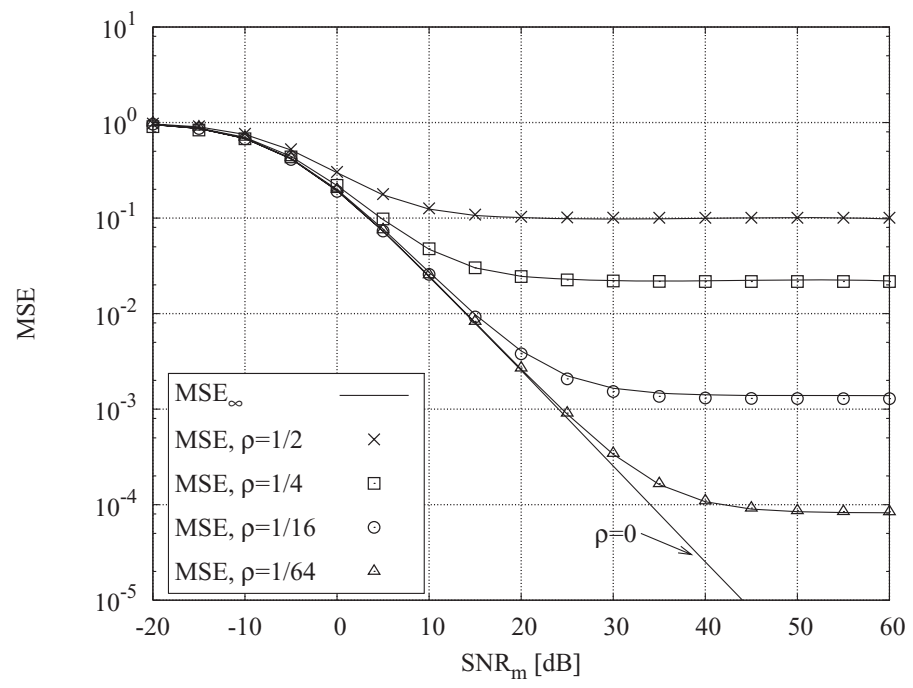

Fig. 3. Uniform distribution: $\mathrm{MSE}_{\infty}$ and $\mathrm{MSE}$ as functions of $\mathrm{SNR}_{m}[\mathrm{~dB}]$ and parameterized by $\rho$, with $\beta=0.2$ and $d=2$.

In this case, the matrix $\mathbf{C}$ is real and, for a constant $\rho=\sigma_{\delta} r^{\prime}$ and for any integer $p$, we have:

$$
\phi\left(\mathbf{C}^{p}\right)=\left(\frac{1}{\sqrt{2 \pi \beta^{\frac{2}{d}} \rho^{2} p}} \operatorname{erf}\left(\pi \beta^{\frac{1}{d}} \rho \sqrt{\frac{p}{2}}\right)\right)^{d} .
$$

The derivation of (19) is reported in Appendix D. In particular, for $\rho \rightarrow+\infty$ (i.e., no knowledge on the network topology) $\phi\left(\mathbf{C}^{p}\right)=0$, while for $\rho=0$ (i.e., perfect knowledge on the topology) $\phi\left(\mathbf{C}^{p}\right)=1$. Also, we have: $\lim _{\beta \rightarrow 0} \phi\left(\mathbf{C}^{p}\right)=1$.

Figure 3 shows the MSE obtained when the network has a Uniform topology distribution and the LMMSE filter in (6) is employed. A Gaussian distribution of the position error $\delta$ is assumed. The solid curves show the asymptotic mean square error $\left(\mathrm{MSE}_{\infty}\right)$ and have been obtained by computing (18), averaging over the eigenvalues of 200 realizations of the matrix $\mathbf{T}$, with $\beta=0.2, d=2$, and $M=6$.

In the plot, the markers represent the empirical average MSE obtained by generating 200 realizations of the measures $\mathbf{p}$ in (2), by computing (4), and by averaging the square error $\|\mathbf{a}-\hat{\mathbf{a}}\|^{2} /(2 M+1)^{d}$, with $\beta=0.2, d=2$, and $M=6$. The curves are parameterized by $\rho$. The plot also shows the asymptotic result for the case $\rho=0$.

Looking at the plot in Figure 3, we notice that, as expected, a better knowledge on the network topology results in a lower MSE. Furthermore, the tight match between asymptotic results and finite system simulation, even for small values of $M$ and $r$, confirms the validity of the asymptotic analysis as well as of the assumption on asymptotic freeness. As a last remark, we mention that very similar results have been obtained for any value of $\beta$. 


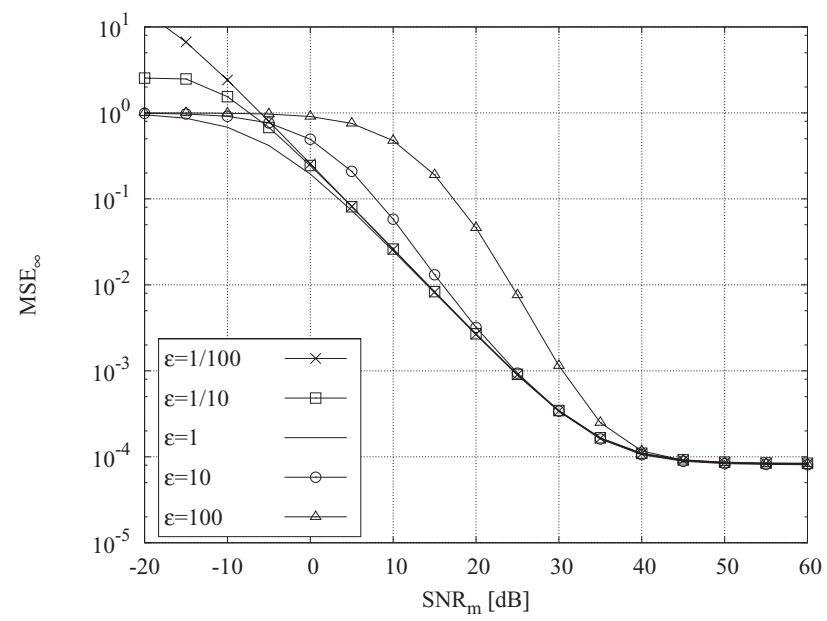

Fig. 4. $\mathrm{MSE}_{\infty}$ for $d=2, \beta=0.2$, and $\rho=1 / 64$, with mismatch in the $\mathrm{SNR}_{m}$ estimation.

In Figure 3, we assumed a perfect knowledge of $\mathrm{SNR}_{m}=1 / \alpha$. However, when $\mathrm{SNR}_{m}$ is unknown and has to be estimated, a mismatch between the estimated and the true value of $\mathrm{SNR}_{m}$ can degrade the system performance in terms of MSE. By writing the estimated $\alpha$ as $\hat{\alpha}=\epsilon \alpha$, with $\epsilon$ being the mismatch coefficient, the LMMSE filter $\mathbf{B}$ in (5) becomes, $\mathbf{B}=\beta \mathbf{G}_{\mathbf{X}}^{\dagger}\left(\mathbf{T}_{\mathbf{X}}+\epsilon \alpha \beta \mathbf{I}\right)^{-1}$ and the expression of $\mathrm{MSE}_{\infty}$ will change accordingly. In Figure 4, we show the impact of the mismatch $\epsilon$ on the system performance, for $d=2, \beta=0.2$, and $\rho=1 / 64$. An overestimate of the $\mathrm{SNR}_{m}$ (i.e., $\epsilon<1$ ) results in a degradation of the performance for low values of $\mathrm{SNR}_{m}$. An underestimate of the $\mathrm{SNR}_{m}$ (i.e., $\epsilon>1$ ) results in a degradation of the MSE for low and moderate values of $\mathrm{SNR}_{m}$. It should be noted, however, that even large mismatches (namely, of one order of magnitude) do not severely affect the performance. We therefore conclude that the system is extremely robust to estimation errors on $\mathrm{SNR}_{m}$.

\subsection{The Uniform-i and Gaussian Topology Distributions}

In the case of distributions other than Uniform, the expression of the asymptotic MSE presented in (18) is no longer valid. Indeed, the results on the matrices $\mathbf{G}$ and $\mathbf{T}$ (namely, equalities (11), (12), and (13)) do not apply. However, below we show that, under certain conditions on $\rho$ and $\beta$, the MSE in the case of Uniform-i and Gaussian layouts can be approximated by (18).

As a first step, let us compute the closed form expression of $\phi\left(\widehat{\mathbf{T}}^{2}\right)$. When $\mathbf{x}$ follows the Uniform distribution, from (11) we have $\phi\left(\widehat{\mathbf{T}}^{2}\right)=\phi\left(\mathbf{T}^{2}\right)$ and Nordio et al. [2006] $\phi\left(\widehat{\mathbf{T}}^{2}\right)=\phi\left(\mathbf{T}^{2}\right)=1+\beta$. In general, instead, when we deal with quasi-equally spaced sensor layouts (as described in Section 4), we have:

$$
\begin{aligned}
& \phi\left(\mathbf{T}^{2}\right)=1+\beta-\beta\left(\int_{\mathcal{A}}\left|C_{\tilde{x}}\left(\mathrm{j} 2 \pi \beta^{\frac{1}{d}}\left(z_{1}-z_{2}\right)\right)\right|^{2} \mathrm{~d} z_{1} \mathrm{~d} z_{2}\right)^{d} \\
& \left.\phi\left(\widehat{\mathbf{T}}^{2}\right)=1+\beta-\beta\left(\int_{\mathcal{A}}\left|C_{\tilde{x}}\left(\mathrm{j} 2 \pi \beta^{\frac{1}{d}}\left(z_{1}-z_{2}\right)\right)\right|^{2}\left|C_{\tilde{\delta}}\left(\mathrm{j} 2 \pi \beta^{\frac{1}{d}}\left(z_{1}-z_{2}\right)\right)\right|^{2} \mathrm{~d} z_{1} \mathrm{~d} z_{2}\right)\right)^{d},
\end{aligned}
$$


where the set $\mathcal{A}$ is defined as $\mathcal{A}=\left\{\left(z_{1}, z_{2}\right) \in \mathbb{R}^{2} \mid z_{1}, z_{2} \in\left[-\frac{1}{2}, \frac{1}{2}\right), z_{1} \neq z_{2}\right\}$ and $\tilde{\delta}=\delta r^{\prime} \sim \mathcal{N}\left(0, \rho^{2}\right)$. The proof can be found in Appendix E.

Specifically, when $x$ follows the Uniform-i distribution, the characteristic functions $C_{\tilde{x}}, C_{\tilde{\delta}}$ are given by $C_{\tilde{x}}\left(\mathrm{j} 2 \pi \beta^{\frac{1}{d}} z\right)=\operatorname{sinc}\left(\beta^{\frac{1}{d}} z\right)$, and $C_{\tilde{\delta}}\left(\mathrm{j} 2 \pi \beta^{\frac{1}{d}} z\right)=$ $\mathrm{e}^{-2 \pi^{2} \beta^{\frac{2}{d}} \rho^{2} z^{2}}$. By using these expressions in (20) and (21), we obtain:

$$
\begin{aligned}
& \phi\left(\mathbf{T}^{2}\right)=1+\beta-\beta\left(\int_{\mathcal{A}} \operatorname{sinc}^{2}\left(\beta^{\frac{1}{d}}\left(z_{1}-z_{2}\right)\right) \mathrm{d} z_{1} \mathrm{~d} z_{2}\right)^{d} \\
& \phi\left(\widehat{\mathbf{T}}^{2}\right)=1+\beta-\beta\left(\int_{\mathcal{A}} \operatorname{sinc}^{2}\left(\beta^{\frac{1}{d}}\left(z_{1}-z_{2}\right)\right) \mathrm{e}^{-4 \pi^{2} \rho^{2} \beta^{\frac{2}{d}}\left(z_{1}-z_{2}\right)^{2}} \mathrm{~d} z_{1} \mathrm{~d} z_{2}\right)^{d} .
\end{aligned}
$$

Instead, when the entries of $\mathbf{x}$ follow the Gaussian distribution, the characteristic function of $\tilde{x}$ becomes: $C_{\tilde{x}}\left(\mathrm{j} 2 \pi \beta^{\frac{1}{d}} \boldsymbol{z}\right)=\mathrm{e}^{-2 \pi^{2} \beta^{\frac{2}{d}} \eta^{2} z^{2}}$ thus,

$$
\begin{aligned}
& \phi\left(\mathbf{T}^{2}\right)=1+\beta-\beta\left(\int_{\mathcal{A}} \mathrm{e}^{\left.-4 \pi^{2} \eta^{2} \beta^{\frac{2}{d}\left(z_{1}-z_{2}\right)^{2}} \mathrm{~d} z_{1} \mathrm{~d} z_{2}\right)^{d}}\right. \\
& \phi\left(\widehat{\mathbf{T}}^{2}\right)=1+\beta-\beta\left(\int_{\mathcal{A}} \mathrm{e}^{-4 \pi^{2} \beta^{\frac{2}{d}}\left(\eta^{2}+\rho^{2}\right)\left(z_{1}-z_{2}\right)^{2}} \mathrm{~d} z_{1} \mathrm{~d} z_{2}\right)^{d} .
\end{aligned}
$$

As is evident by comparing (20) with (21), the approximation $\phi\left(\widehat{\mathbf{T}}^{2}\right) \approx$ $\phi\left(\mathbf{T}^{2}\right)$ is tight when $\left|C_{\tilde{\delta}}\left(\mathrm{j} 2 \pi \beta^{\frac{1}{d}}\left(z_{1}-z_{2}\right)\right)\right|^{2} \approx 1$. More specifically, in the case where $\delta$ is Gaussian distributed, the approximation is tight when $\exp \left(-4 \pi^{2} \beta^{\frac{2}{d}} \rho^{2}\left(z_{1}-z_{2}\right)^{2}\right) \approx 1$, that is, for

$$
\rho \ll 1 /\left(2 \pi \beta^{\frac{1}{d}}\right) .
$$

We observe that, under this condition, also the other results derived for the uniform sensor deployment can be applied, that is, the approximations $\phi\left(\widehat{\mathbf{T}}^{p}\right) \approx$ $\phi\left(\mathbf{T}^{p}\right), \phi\left(\mathbf{G} \widehat{\mathbf{G}}^{\dagger} \widehat{\mathbf{T}}^{p}\right) \approx \phi\left(\widehat{\mathbf{G G}}^{\dagger} \mathbf{T}^{p}\right)$ and $\phi\left(\mathbf{G} \widehat{\mathbf{G}}^{\dagger} \widehat{\mathbf{T}}^{p} \widehat{\mathbf{G}}^{\dagger}\right) \approx \phi\left(\widehat{\mathbf{G G}}^{\dagger} \mathbf{T}^{p} \mathbf{G} \widehat{\mathbf{G}}^{\dagger}\right)$ are tight.

We therefore conclude that under the condition in (22) the asymptotic MSE in the case of Uniform-i and Gaussian sensor layouts can be approximated by (18), with the appropriate distribution of $\lambda$.

Figure 5 shows histograms of $f_{\lambda}(z, \beta, 2)$ for $\beta=0.2,0.3$, and 0.4 , when the entries of $\mathbf{x}$ follow the Uniform-i (solid lines) and Gaussian (dashed lines) distributions. The results have been obtained by computing the eigenvalues of realizations of $\mathbf{T}$, with $M=6$; the bin width is 0.1 . For the Gaussian distribution, we set $\eta^{2}=\frac{1}{12}$ so as to obtain the same variance as in the Uniform-i case. The histogram of the two distributions of $\lambda$ look very similar. They are concentrated around $z=1$ for low values of $\beta$. As $\beta$ increases, the distribution becomes more spread and the probability of small eigenvalues increases. However, while for $\beta=0.4$ these distributions do not show a significant amount of small eigenvalues, as depicted in Figure 2, the Uniform distribution shows a significant fraction of eigenvalues close to zero already for $\beta=0.35$. The reason for such different behavior is that distributions Uniform-i and Gaussian guarantee a more regular sensor deployment; recall that a perfectly regular (equally spaced) node deployment results in $\mathbf{T}=\mathbf{I}$ and $f_{\lambda}(z, \beta, d)=\delta_{\mathrm{D}}(z-1)$. For example, a realization of $\mathbf{X}$ such that $x_{m, q}<0,(m=1, \ldots, d, q=1, \ldots, r)$ 


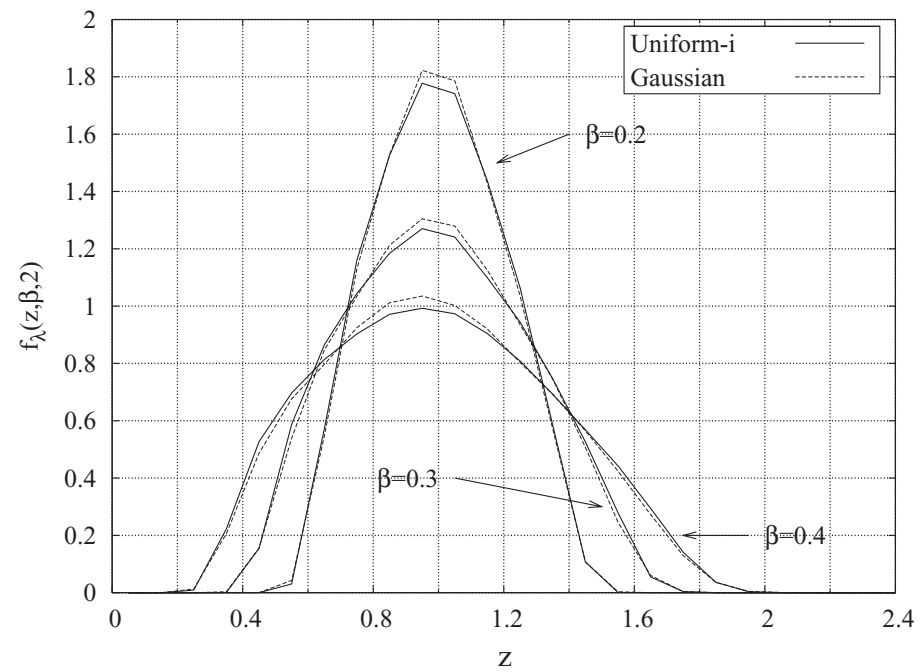

Fig. 5. Histograms of $f_{\lambda}(z, \beta, 2)$, for Uniform-i and Gaussian distributions and different values of $\beta$.

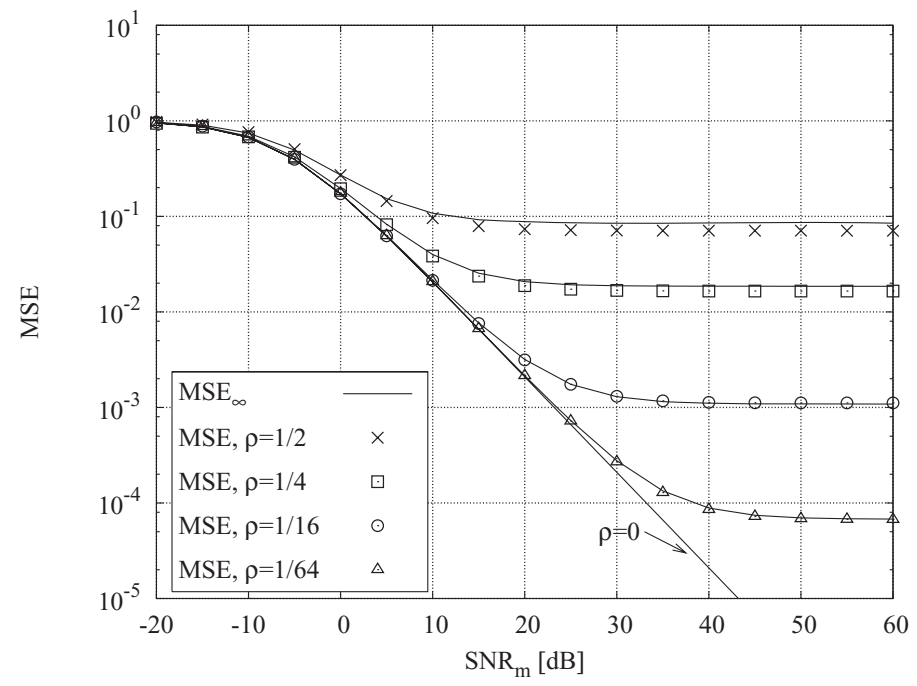

Fig. 6. Uniform-i distribution: $\mathrm{MSE}_{\infty}$ and $\mathrm{MSE}$ as functions of $\mathrm{SNR}_{m}$ and for different values of $\rho, \beta=0.2$, and $d=2$.

is likely to happen for the Uniform distribution, while it is impossible for the Uniform-i distribution, and very unlikely in the case of Gaussian distribution. Unfortunately, also for these simple network topologies a closed form expression of $f_{\lambda}(z, \beta, d)$ is unknown, and therefore (18) must be computed numerically.

Figure 6 shows the MSE obtained when the network topology follows the Uniform-i distribution, in the case of perfect $\mathrm{SNR}_{m}$ knowledge. We used the same parameter setting as in Figure 3 . Here, $\mathrm{MSE}_{\infty}$ has been attained through (18), which is an approximation of the true asymptotic MSE. As for Figure 3, the 


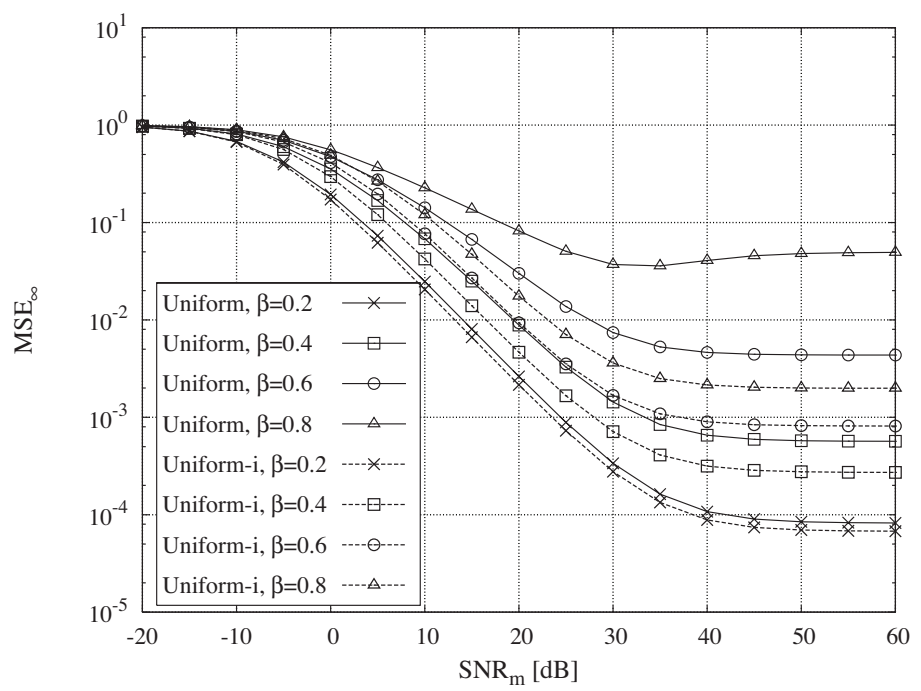

Fig. 7. $\mathrm{MSE}_{\infty}$ achieved by the Uniform and Uniform-i distributions for $\beta=0.2,0.4,0.6,0.8, \rho=$ $1 / 64$, and $d=2$.

markers represent the empirical MSE obtained by generating 200 realizations of the measures $\mathbf{p}$ as in (2), by computing (4), and by averaging the square error $\|\mathbf{a}-\hat{\mathbf{a}}\|^{2} /(2 M+1)^{d}$.

Recall that the condition that guarantees a tight approximation is $\rho \ll$ $1 /\left(2 \pi \beta^{\frac{1}{d}}\right)$, i.e., in our case: $\rho \ll 0.36$. Indeed, we observe that the match between the numerical evaluation of the MSE as in (3) and the results computed through (18) is perfect for $\rho=1 / 64,1 / 16$, while the results are quite close for higher $\rho$ 's.

Figure 7 compares the $\mathrm{MSE}_{\infty}$ achieved under the Uniform and Uniform-i distributions, with a perfect $\mathrm{SNR}_{m}$ knowledge, for $\rho=1 / 64$ and various values of $\beta$. The curves have been computed using (18) for both distributions, since the condition $\rho=1 / 64 \ll 1 /\left(2 \pi \beta^{\frac{1}{d}}\right)$ is satisfied. In general, for any given $\beta$, the Uniform-i distribution provides a lower $\mathrm{MSE}_{\infty}$, especially for high values of $\mathrm{SNR}_{m}$.

Figure 8 shows the $\mathrm{MSE}_{\infty}$ achieved by the Gaussian topology distribution, perfect $\mathrm{SNR}_{m}$ knowledge, for $\beta=0.4, \rho=1 / 64$ [Liu et al. 2006], and $\eta^{2}=$ $0,1 / 12,1,10$. For the same values of $\beta$ and $\rho$, the Uniform-i distribution gives very similar results to those obtained with the Gaussian distribution when $\eta^{2}=1 / 12$. As expected, lower values of $\eta^{2}$ result in a more equally spaced deployment and, thus, in a lower $\mathrm{MSE}_{\infty}$. Note that $\eta^{2}=0$ results in a perfectly equally spaced deployment of the sensors. The floor shown by the MSE curve for high $\mathrm{SNR}_{m}$ is due to the imperfect knowledge of $\mathbf{x}$, that is, to the variance of the position error $\delta$. Also, we observe that the difference between the performance of an equally spaced deployment $\left(\eta^{2}=0\right)$ and the performance of a Gaussian deployment with $\eta^{2}=1 / 12$ is negligible. The above considerations hold for any value of $\beta$. 


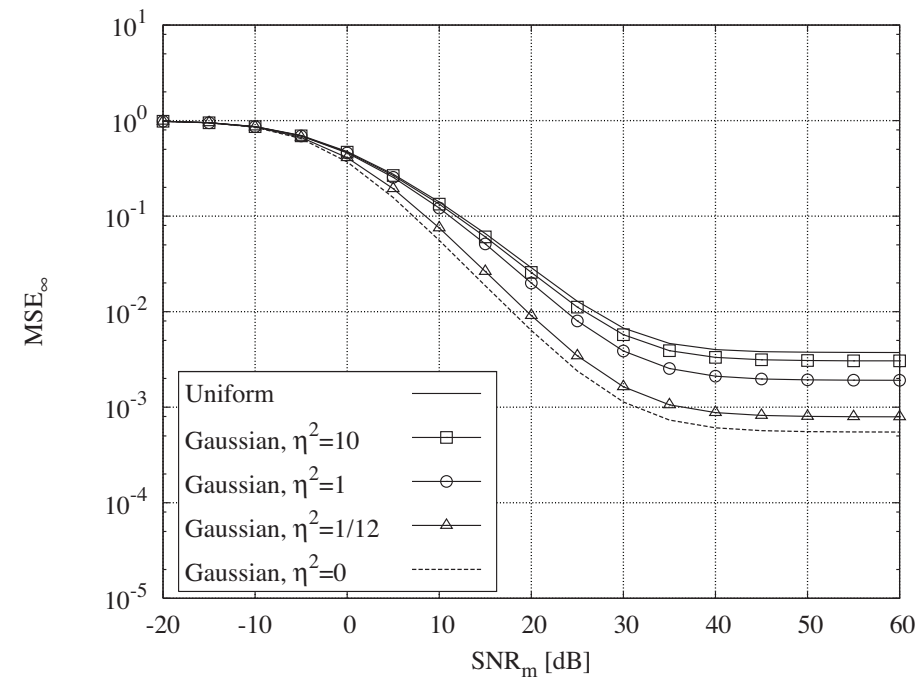

Fig. 8. $\mathrm{MSE}_{\infty}$ achieved by the Gaussian topology distribution for $\beta=0.4, d=2, \rho=1 / 64$, and $\eta^{2}=0,1 / 12,1,10$

Below, we provide an example that shows how the results we obtained can be used for the design of sensor networks.

Example. A network characterized by $\beta=0.4, \rho=1 / 64$, and $\mathrm{SNR}_{m}=$ $30 \mathrm{~dB}$ is deployed by an airplane throwing sensors at equally spaced time instants. The unpredictable effects of winds result in a non-perfectly equallyspaced deployment on the ground. The distribution of the deployment error is assumed to be Gaussian with parameter $\eta^{2}$. We want to determine how accurate the sensor deployment needs to be, that is, the maximum $\eta^{2}$ for which the network provides an MSE not larger than $2 \cdot 10^{-3}$.

By looking at Figure 8, we observe that, for $\mathrm{SNR}_{m}=30 \mathrm{~dB}, \eta^{2}<1 / 12$ is enough to provide the required MSE. It is interesting to notice that a perfectly equally-spaced (and very expensive) deployment, that is, $\eta^{2}=0$, does not provide an MSE lower than $10^{-3}$.

\subsection{A Measure of Sensor Layout Regularity}

Here, we introduce a new parameter, $\tau(0 \leq \tau \leq 1)$, which provides a measure of the regularity of the sensors deployment.

In general, we observe that a more equally spaced distribution results in a lower variance of the eigenvalues of $\mathbf{T}$, given by $\sigma_{\lambda}^{2}=\mathbb{E}\left[\lambda^{2}\right]-\mathbb{E}[\lambda]^{2}=\phi\left(\mathbf{T}^{2}\right)-$ $\phi(\mathbf{T})^{2}$. Since $\phi(\mathbf{T})=1$ for any distribution of $x$ [Nordio et al. 2006], using (20) we can write:

$$
\sigma_{\lambda}^{2}=\beta-\beta\left(\int_{\mathcal{A}}\left|C_{\tilde{x}}\left(\mathrm{j} 2 \pi \beta^{\frac{1}{d}}\left(z_{1}-z_{2}\right)\right)\right|^{2} \mathrm{~d} z_{1} \mathrm{~d} z_{2}\right)^{d}
$$

By defining $\tau=\left(\int_{\mathcal{A}}\left|C_{\tilde{x}}\left(\mathrm{j} 2 \pi \beta^{\frac{1}{d}}\left(z_{1}-z_{2}\right)\right)\right|^{2} \mathrm{~d} z_{1} \mathrm{~d} z_{2}\right)^{d}$ we have $\sigma_{\lambda}^{2}=\beta(1-\tau)$. Note that a perfectly equally-spaced deployment is characterized by $f_{\tilde{x}}=\delta_{\mathrm{D}}(z)$, that 
is, $C_{\tilde{x}}(s)=1$, and, by consequence, $\tau=1$ and $\sigma_{\lambda}^{2}=0$. For the Uniform distribution, instead, we have $\tau=0$ and $\sigma_{\lambda}^{2}=\beta$ [Nordio et al. 2006]. We therefore expect that, given $\beta$ and $d$, the more equally-spaced the sensor layout is, the higher the value of $\tau$. In our case study of Uniform-i layout, the parameter $\tau$ is given by:

$$
\tau=\frac{\left(2 \cos ^{2}\left(\pi \beta^{\frac{1}{d}}\right)-2+\operatorname{Ci}\left(2 \pi \beta^{\frac{1}{d}}\right)-\ln \left(2 \pi \beta^{\frac{1}{d}}\right)-\gamma+2 \operatorname{Si}\left(2 \pi \beta^{\frac{1}{d}}\right) \pi \beta^{\frac{1}{d}}\right)^{d}}{\pi^{2 d} \beta^{2}}
$$

while for the Gaussian distribution we have:

$$
\tau=\left(\frac{\operatorname{erf}\left(2 \pi \beta^{\frac{1}{d}} \eta\right)}{2 \sqrt{\pi} \beta^{\frac{1}{d}} \eta}-\frac{1-\exp \left(-4 \pi^{2} \beta^{\frac{2}{d}} \eta^{2}\right)}{4 \pi^{2} \beta^{\frac{2}{d}} \eta^{2}}\right)^{d}
$$

The functions $\mathrm{Ci}(\cdot)$ and $\mathrm{Si}(\cdot)$, and the constant $\gamma$ are the cosine integral, the sine integral and the Euler-Mascheroni constant [Weisstein 2008], respectively. As an example, for $d=2, \beta=0.4$ and $\eta^{2}=1 / 12$ (i.e., Uniform-i and Gaussian distributions with the same variance), we have $\tau=0.673$ for the Uniform-i and $\tau=0.684$ for the Gaussian.

\subsection{Closed Form Approximation for $d=2$}

In this section, we focus on the case $d=2$, which is of great practical interest, and derive a closed form approximation of (18) that holds for the Uniform distribution, as well as for the Uniform-i and Gaussian layouts under the condition in (22). To obtain such an approximation, a closed form approximation of $f_{\lambda}(z, \beta, d)$ is required.

Figure 5 shows that, for the Uniform-i and Gaussian network topologies, the shape of the empirical pdf of $\lambda$ can be approximated by a parabolic pdf $f_{p}(z, \beta)$, with mean $\mu_{p}$ and variance $\sigma_{p}^{2}$. To match the distribution of the eigenvalues shown in Figure 5 with the parabolic pdf, we set $\sigma_{p}^{2}=\sigma_{\lambda}^{2}$ and $\mu_{p}=\mathbb{E}[\lambda]=1$. Thus, we define $f_{p}(z, \beta)$ as

$$
f_{p}(z, \beta)=-\frac{3 \sqrt{5}}{100 \sigma_{\lambda}^{3}}\left(z^{2}-2 z+1-5 \sigma_{\lambda}^{2}\right)
$$

for $1-\sqrt{5 \sigma_{\lambda}^{2}}<z<1+\sqrt{5 \sigma_{\lambda}^{2}}$ and 0 elsewhere. Moreover, we require that $1-\sqrt{5 \sigma_{\lambda}^{2}}>0$, that is, $\sigma_{\lambda}^{2}<1 / 5$, in order to avoid that the support of $f_{p}(z, \beta)$ lies on the negative $z$ axis. Notice that the dependence of $f_{p}(z, \beta)$ on $\beta$ is evident from the expression of $\sigma_{\lambda}^{2}$ in (23).

Figure 9 shows the histograms of $f_{\lambda}(z, \beta, 2)$ for the Uniform-i distribution (solid lines) and its approximation given by the parabolic pdf (dashed lines), for $\beta=0.2,0.3$ and 0.4 . The bin width is 0.1 .

Note, however, that our goal here is to find a closed form approximation of the asymptotic MSE, we are therefore interested in assessing the approximation accuracy for the MSE rather than for the pdf. To compute the approximate expression of the $\mathrm{MSE}_{\infty}$, we use the parabolic pdf in (18), thus obtaining integrals of the type $\int \frac{z^{n}}{(z+\alpha \beta)^{m}} \mathrm{~d} z$ for $m=1,2$ and $n=1,2,3$, 4. Such integrals have 


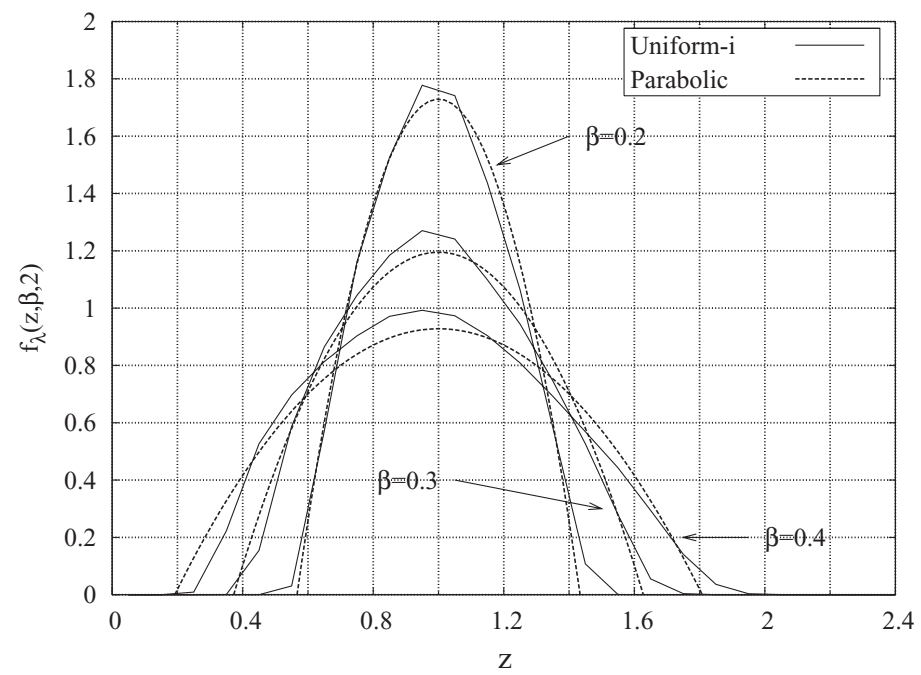

Fig. 9. Histogram of $f_{\lambda}(z, \beta, 2)$ and parabolic approximation, in the case of Uniform-i and $\beta=$ $0.2,0.3,0.4$.

a closed form expression, since they are computed over a finite domain and their arguments are rational functions without poles in the integration domain. The resulting expression of $\mathrm{MSE}_{\infty}$ is quite complicate and is omitted here. However, we point out that $\mathrm{MSE}_{\infty}$ is an increasing function in $\alpha$, and $\beta$, and a decreasing function in the parameter $\tau$. When the Gaussian topology is considered, $\mathrm{MSE}_{\infty}$ also depends on the standard deviation $\eta$, through the expression of $\tau$ given in Section 5.3.

For the Uniform-i distribution, Figure 10 compares the asymptotic MSE given by the parabolic approximation, that is, $\lambda$ distributed as in (24) (dashed lines), and the $\mathrm{MSE}_{\infty}$ given by (18) (points) with perfect $\mathrm{SNR}_{m}$ knowledge, for $d=2, \rho=1 / 64$, and different values of $\beta$. We observe an excellent match, for all considered values of $\beta$ and $\mathrm{SNR}_{m}$.

Similarly, Figure 11 compares the asymptotic MSE and its closed form approximation for the case of Gaussian distribution. Here, we set: $d=2$, $\eta^{2}=1 / 12, \beta=0.3$, and $\rho=1 / 64$. Again, we obtain an excellent match.

\section{CONCLUSIONS}

We considered a sensor network whose nodes sample a bandlimited scalar field defined over a multidimensional domain. The field is then reconstructed at a central controller by using a processing technique based on linear filtering. We assumed that the transmission of the samples from the sensors to the central controller is always successful and that the sensors locations are randomly distributed over the observed area; in particular, we focused on quasi-equally spaced layouts of the sensors. Furthermore, we assumed the reconstruction algorithm to have imperfect knowledge on the network topology. 


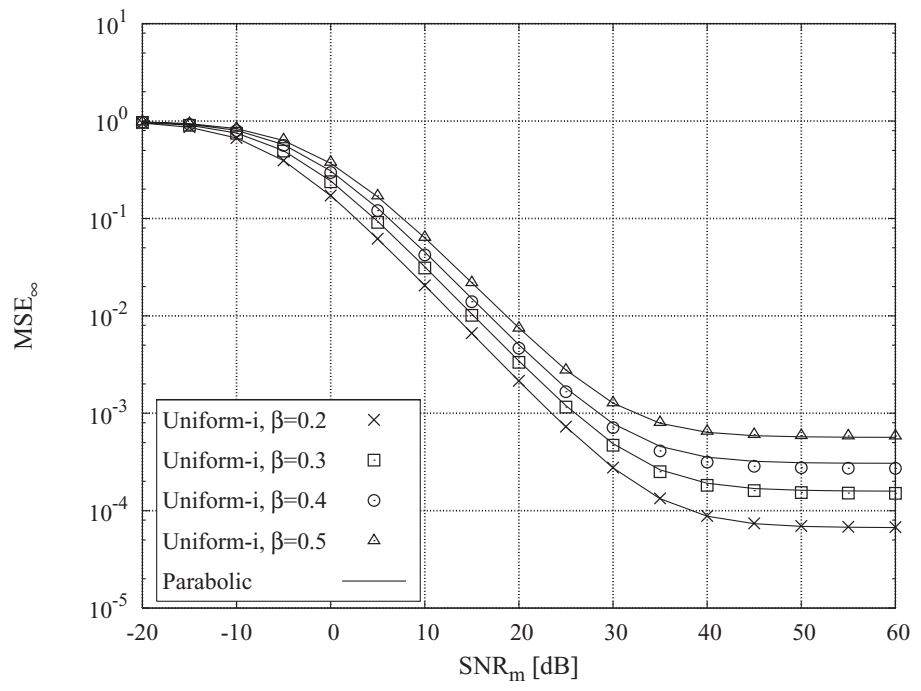

Fig. 10. Uniform-i distribution: $\mathrm{MSE}_{\infty}$ given by (18) and its parabolic approximation, for $d=2$, $\rho=1 / 64$ and $\beta=0.2,0.3,0.4,0.5$.

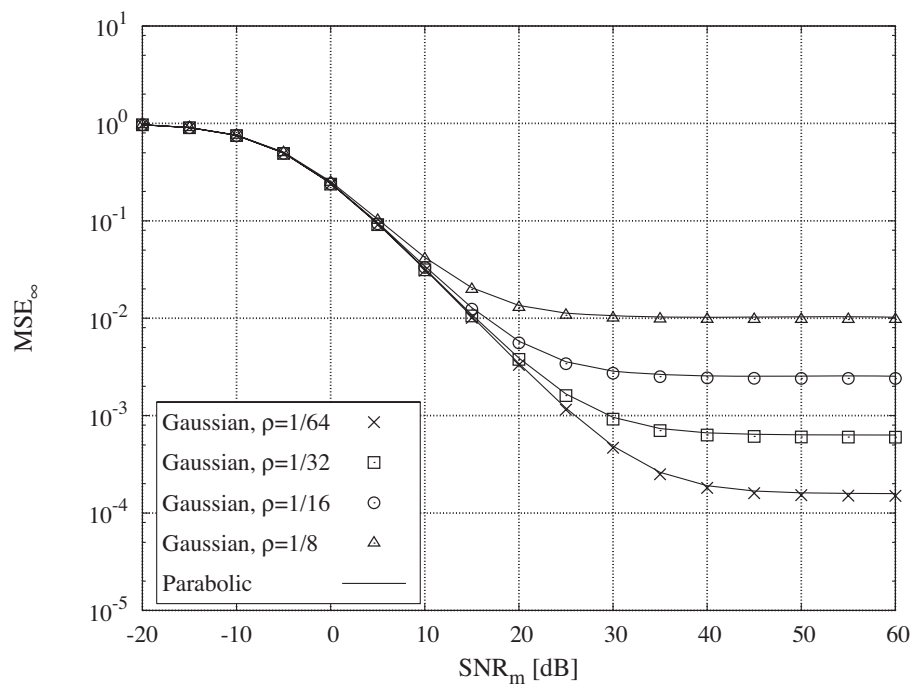

Fig. 11. Gaussian distribution: $\mathrm{MSE}_{\infty}$ given by (18) and its parabolic approximation, for $d=2$, $\beta=0.3, \eta^{2}=1 / 12$, and different values of $\rho$.

We showed via both analysis and simulation how the performance of the reconstruction technique depends on the distribution of the sensors in the area of interest. As case study, we considered three network topology distributions, and we compared their performance in terms of the MSE on the reconstructed field. We identified a parameter, $\tau$, that provides a measure of the regularity of the sensor deployment. Also, in the case of a bidimensional domain, we derived 
a simple analytical approximation of the MSE, which showed to be very tight when compared to simulation results.

\section{APPENDIXES}

\section{A. LIST OF PARAMETERS}

$r \quad$ Number of samples delivered to the sink (or, number of sensors in the network)

$M \quad$ Approximate one-sided bandwidth per dimension of the field

$d \quad$ Dimension of the field domain

$r^{\prime} \quad$ Number of samples per dimension

$\beta \quad$ Ratio of the double-sided bandwidth of the field to the number of sensors

$\alpha \quad$ Inverse of the signal-to-noise ration on the sensor measures

$\Delta \quad$ Matrix representing the estimation error on the sensor locations

$\rho \quad$ Measure of the uncertainty on the network topology

X Matrix collecting the sampling positions

a The field discrete spectrum

p Vector collecting the sensor measures

n Vector denoting the noise affecting the sensor measures

$\mathbf{G} \quad(2 M+1)^{d} \times r$ matrix with $\mathbf{G}_{v(\ell), q}=\frac{1}{\sqrt{(2 M+1)^{d}}} \mathrm{e}^{-\mathrm{j} 2 \pi \ell^{\mathrm{T}} \mathbf{x}_{q}}$

$\mathbf{T} \quad \mathbf{T}=\beta \mathbf{G G}^{\dagger}$

$C_{x}(s) \quad$ Characteristic function of $x$

\section{B. PROOFS OF (11), (12), AND (13)}

Here, we provide the proof of claims (11), (12), and (13). With regards to equation (11), from the definition of $\phi(\cdot)$, we have:

$$
\phi\left(\widehat{\mathbf{T}}^{p}\right)=\lim _{M, r \rightarrow+\infty} \frac{1}{(2 M+1)^{d}} \mathbb{E}\left[\operatorname{Tr}\left\{\widehat{\mathbf{T}}^{p}\right\}\right] .
$$

By expanding the matrix power, we obtain:

$$
\begin{aligned}
\mathbb{E}\left[\operatorname{Tr}\left\{\mathbf{T}^{p}\right\}\right] & =\mathbb{E}\left[\sum_{\ell_{1}}\left(\mathbf{T}^{p}\right)_{v\left(\ell_{1}\right), v\left(\ell_{1}\right)}\right] \\
& =\mathbb{E}\left[\sum_{\mathbf{L}}(\mathbf{T})_{v\left(\ell_{1}\right), v\left(\ell_{2}\right)}(\mathbf{T})_{v\left(\ell_{2}\right), v\left(\ell_{3}\right)} \cdots(\mathbf{T})_{v\left(\ell_{p-1}\right), v\left(\ell_{p}\right)}(\mathbf{T})_{v\left(\ell_{p}\right), v\left(\ell_{1}\right)}\right] .
\end{aligned}
$$

where $\mathbf{L}=\left[\ell_{1}, \ldots, \ell_{p}\right]$ is $d \times p$ matrix of integers with entries ranging in $-M, \ldots,+M$. Since $\mathbf{T}=\beta \mathbf{G G}^{\dagger}$, the $\left\{v(\ell), v\left(\ell^{\prime}\right)\right\}$-th entry of $\mathbf{T}$ is given by: $(\mathbf{T})_{v(\ell), v\left(\ell^{\prime}\right)}=\frac{1}{r} \sum_{q=1}^{r} \mathrm{e}^{-\mathrm{j} 2 \pi\left(\ell-\ell^{\prime}\right)^{\mathrm{T}} \mathbf{x}_{q}}$. Therefore,

$$
\mathbb{E}\left[\operatorname{Tr}\left\{\mathbf{T}^{p}\right\}\right]=\frac{1}{r^{p}} \sum_{\mathbf{L}} \sum_{\mathbf{q}} \mathbb{E}\left[\mathrm{e}^{-\mathrm{j} 2 \pi\left(\ell_{1}-\ell_{2}\right)^{\mathrm{T}} \mathbf{x}_{q_{1}}-\cdots-\mathrm{j} 2 \pi\left(\ell_{p}-\ell_{1}\right)^{\mathrm{T}} \mathbf{x}_{q_{p}}}\right] .
$$

ACM Transactions on Sensor Networks, Vol. 6, No. 2, Article 11, Publication date: February 2010. 
where $\mathbf{q}=\left[q_{1}, \ldots, q_{p}\right]$ is a vector of integers with $q_{i}=1, \ldots, r$. Similarly,

$$
\begin{aligned}
& \mathbb{E}\left[\operatorname{Tr}\left\{\widehat{\mathbf{T}}^{p}\right\}\right]= \frac{1}{r^{p}} \sum_{\mathbf{L}} \sum_{\mathbf{q}} \mathbb{E}\left[\mathrm{e}^{\left.-\mathrm{j} 2 \pi\left(\ell_{1}-\ell_{2}\right)^{\mathrm{T}} \hat{\mathbf{x}}_{q_{1}}-\cdots-\mathrm{j} 2 \pi\left(\ell_{p}-\ell_{1}\right)^{\mathrm{T}} \hat{\mathbf{x}}_{q_{p}}\right]}\right. \\
&= \frac{1}{r^{p}} \sum_{\mathbf{L}} \sum_{\mathbf{q}} \underset{\mathbf{X}}{\mathbb{E}}\left[\mathrm{e}^{\left.-\mathrm{j} 2 \pi\left(\ell_{1}-\ell_{2}\right)^{\mathrm{T}} \mathbf{x}_{q_{1}}-\cdots-\mathrm{j} 2 \pi\left(\ell_{p}-\ell_{1}\right)^{\mathrm{T}} \mathbf{x}_{q_{p}}\right]}\right. \\
& \cdot \underset{\Delta}{\mathbb{E}}\left[\mathrm{e}^{\left.-\mathrm{j} 2 \pi\left(\ell_{1}-\ell_{2}\right)^{\mathrm{T}} \delta_{q_{1}}-\cdots-\mathrm{j} 2 \pi\left(\ell_{p}-\ell_{1}\right)^{\mathrm{T}} \delta_{q_{p}}\right]}\right. \\
&=\frac{1}{r^{p}} \sum_{\mathbf{L}} \sum_{\mathbf{q}} \underset{\mathbf{x}}{\mathbb{E}}\left[\prod_{m=1}^{d} \mathrm{e}^{-\mathrm{j} 2 \pi\left(\ell_{m, 1}-\ell_{m, 2}\right) x_{m, q_{1}}-\cdots-\mathrm{j} 2 \pi\left(\ell_{m, p}-\ell_{m, 1}\right) x_{m, q_{p}}}\right] \\
& \cdot \underset{\Delta}{\mathbb{E}}\left[\prod_{m=1}^{d} \mathrm{e}^{-\mathrm{j} 2 \pi\left(\ell_{m, 1}-\ell_{m, 2}\right) \delta_{m, q_{1}}-\cdots-\mathrm{j} 2 \pi\left(\ell_{m, p}-\ell_{m, 1}\right) \delta_{m, q_{p}}}\right] .
\end{aligned}
$$

In practice,for any integer $\ell$, (25) is obtained by summing products of averages of the type $\underset{x}{\mathbb{E}}\left[\mathrm{e}^{-\mathrm{j} 2 \pi \ell x}\right] \underset{\delta}{\mathbb{E}}\left[\mathrm{e}^{-\mathrm{j} 2 \pi \ell \delta}\right]$. Notice that: $\underset{x}{\mathbb{E}}\left[\mathrm{e}^{-\mathrm{j} 2 \pi \ell x}\right] \underset{\delta}{\mathbb{E}}\left[\mathrm{e}^{-\mathrm{j} 2 \pi \ell \delta}\right]=$ $C_{x}(-\mathrm{j} 2 \pi \ell) C_{\delta}(-\mathrm{j} 2 \pi \ell)$, where $C_{x}(s)$ and $C_{\delta}(s)$ are the characteristic functions of the random variables $x$ and $\delta$, respectively. Since $x$ is uniformly distributed in $[-1 / 2,+1 / 2)$, then $C_{x}(s)=\int_{-1 / 2}^{+1 / 2} \mathrm{e}^{s x} \mathrm{~d} x=\left(\mathrm{e}^{s / 2}-\mathrm{e}^{-s / 2}\right) / s$. Thus, $C_{x}(-\mathrm{j} 2 \pi \ell)=1$ for $\ell=0$ and $C_{x}(-\mathrm{j} 2 \pi \ell)=0$ for $\ell \neq 0$. Taking into account that $C_{\delta}(0)=1$ by definition of the characteristic function, it follows that:

$$
C_{x}(-\mathrm{j} 2 \pi \ell) C_{\delta}(-\mathrm{j} 2 \pi \ell)=C_{x}(-\mathrm{j} 2 \pi \ell) C_{\delta}(0)=C_{x}(-\mathrm{j} 2 \pi \ell) .
$$

It turns out that: $\underset{x}{\mathbb{E}}\left[\mathrm{e}^{-\mathrm{j} 2 \pi \ell x}\right] \underset{\delta}{\mathbb{E}}\left[\mathrm{e}^{-\mathrm{j} 2 \pi \ell \delta}\right]=\mathbb{E}\left[\mathrm{e}^{-\mathrm{j} 2 \pi \ell x}\right]$ and, by consequence, $\mathbb{E}\left[\operatorname{Tr}\left\{\widehat{\mathbf{T}}^{p}\right\}\right]=\mathbb{E}\left[\operatorname{Tr}\left\{\mathbf{T}^{p}\right\}\right]$. We finally obtain that $\phi\left(\widehat{\mathbf{T}}^{p}\right)=\phi\left(\mathbf{T}^{p}\right)$.

The proof of (12) can be given in a similar way. Since the $\left\{v\left(\ell^{\prime}\right), v(\ell)\right\}$-th element of $\mathbf{G} \widehat{\mathbf{G}}^{\dagger}$ can be written as $\left(\mathbf{G} \widehat{\mathbf{G}}^{\dagger}\right)_{\nu\left(\ell^{\prime}\right), \nu(\ell)}=(2 M+1)^{-d} \sum_{q^{\prime}=1}^{r} \mathrm{e}^{-\mathrm{j} 2 \pi\left(\ell^{\prime \mathrm{T}} \mathbf{x}_{q^{\prime}}-\ell^{\mathrm{T}} \hat{\mathbf{x}}_{q^{\prime}}\right)}$ using the above notation, we obtain:

$$
\begin{aligned}
& \mathbb{E}\left[\operatorname{Tr}\left\{\mathbf{G} \widehat{\mathbf{G}}^{\dagger} \widehat{\mathbf{T}}^{p}\right\}\right]=\mathbb{E}\left[\sum_{\ell^{\prime}} \sum_{\ell}\left(\mathbf{G} \widehat{\mathbf{G}}^{\dagger}\right)_{\nu\left(\ell^{\prime}\right), \nu(\ell)}\left(\widehat{\mathbf{T}}^{p}\right)_{\nu(\ell), \nu\left(\ell^{\prime}\right)}\right] \\
& =\frac{1}{(2 M+1)^{d} r^{p}} \sum_{\ell^{\prime}, q^{\prime}, \mathbf{L}, \mathbf{q}} \mathbb{E}\left[\mathrm{e}^{-\mathrm{j} 2 \pi\left(\ell^{\prime \mathrm{T}} \mathbf{x}_{q^{\prime}}-\ell_{1}^{\mathrm{T}} \hat{\mathbf{x}}_{q^{\prime}}\right)} \mathrm{e}^{\left.-\mathrm{j} 2 \pi\left(\ell_{1}-\ell_{2}\right)^{\mathrm{T}} \hat{\mathbf{x}}_{q_{1}}-\ldots-\mathrm{j} 2 \pi\left(\ell_{p}-\ell^{\prime}\right)^{\mathrm{T}} \hat{\mathbf{x}}_{q_{p}}\right] .}\right.
\end{aligned}
$$

Notice that: $\ell^{\prime \mathrm{T}} \mathbf{x}_{q^{\prime}}-\ell_{1}^{\mathrm{T}} \hat{\mathbf{x}}_{q^{\prime}}=\ell^{\prime \mathrm{T}}\left(\hat{\mathbf{x}}_{q^{\prime}}-\boldsymbol{\delta}_{q^{\prime}}\right)-\ell_{1}^{\mathrm{T}} \hat{\mathbf{x}}_{q^{\prime}}=\left(\boldsymbol{\ell}^{\prime}-\boldsymbol{\ell}_{1}\right)^{\mathrm{T}} \hat{\mathbf{x}}_{q^{\prime}}-\ell^{\prime \mathrm{T}} \boldsymbol{\delta}_{q^{\prime}}$ thus,

$$
\mathbb{E}\left[\operatorname{Tr}\left\{\mathbf{G} \widehat{\mathbf{G}}^{\dagger} \widehat{\mathbf{T}}^{p}\right\}\right]=\sum_{\ell^{\prime}, q^{\prime}, \mathbf{L}, \mathbf{q}} \frac{\mathbb{E}\left[\mathrm{e}^{\mathrm{j} 2 \pi\left(\ell^{\prime T} \delta_{q^{\prime}}-\left(\ell^{\prime}-\ell_{1}\right)^{\mathrm{T}} \hat{\mathbf{x}}_{q^{\prime}}-\left(\ell_{1}-\ell_{2}\right)^{\mathrm{T}} \hat{\mathbf{x}}_{q_{1}}-\ldots-\left(\ell_{p}-\ell^{\prime}\right)^{\mathrm{T}} \hat{\mathbf{x}}_{q_{p}}\right]}\right.}{(2 M+1)^{d} r^{p}},
$$

whose computation involves sums of products of averages where each factor is of the type $\underset{x}{\mathbb{E}}\left[\mathrm{e}^{-\mathrm{j} 2 \pi \ell x}\right] \underset{\delta}{\mathbb{E}}\left[\mathrm{e}^{-\mathrm{j} 2 \pi \ell \delta}\right] \underset{\delta}{\mathbb{E}}\left[\mathrm{e}^{\mathrm{j} 2 \pi \ell^{\prime} \delta}\right]$ or of the type $\underset{x}{\mathbb{E}}\left[\mathrm{e}^{-\mathrm{j} 2 \pi \ell x}\right] \underset{\delta}{\mathbb{E}}\left[\mathrm{e}^{-\mathrm{j} 2 \pi\left(\ell-\ell^{\prime}\right) \delta}\right]$, for any integers $\ell, \ell^{\prime}$. In both cases, because of the above mentioned properties of 
$C_{x}(-\mathrm{j} 2 \pi \ell)$, the result is: $\underset{x}{\mathbb{E}}\left[\mathrm{e}^{-\mathrm{j} 2 \pi \ell x}\right] \underset{\delta}{\mathbb{E}}\left[\mathrm{e}^{\mathrm{j} 2 \pi \ell^{\prime} \delta}\right]$ and

$$
\mathbb{E}\left[\operatorname{Tr}\left\{\mathbf{G} \widehat{\mathbf{G}}^{\dagger} \widehat{\mathbf{T}}^{p}\right\}\right]=\sum_{\ell^{\prime}, q^{\prime}, \mathbf{L}, \mathbf{q}} \frac{\mathbb{E}\left[\mathrm{e}^{\mathrm{j} 2 \pi\left(\ell^{\mathrm{T}} \delta_{q^{\prime}}-\left(\ell^{\prime}-\ell_{1}\right)^{\mathrm{T}} \mathbf{x}_{q^{\prime}}-\left(\ell_{1}-\ell_{2}\right)^{\mathrm{T}} \mathbf{x}_{q_{1}}-\ldots-\left(\ell_{p}-\ell^{\prime}\right)^{\mathrm{T}} \mathbf{x}_{q_{p}}\right)}\right]}{(2 M+1)^{d} r^{p}} .
$$

Being the $\left(\widehat{\mathbf{G}} \mathbf{G}^{\dagger}\right)_{v\left(\ell^{\prime}\right), v(\ell)}=(2 M+1)^{-d} \sum_{q^{\prime}=1}^{r} \mathrm{e}^{-\mathrm{j} 2 \pi\left(\ell^{\mathrm{T}} \hat{\mathbf{x}}_{q^{\prime}}-\ell^{\mathrm{T}} \mathbf{x}_{q^{\prime}}\right)}$ then

$$
\mathbb{E}\left[\operatorname{Tr}\left\{\widehat{\mathbf{G}} \mathbf{G}^{\dagger} \widehat{\mathbf{T}}^{p}\right\}\right]=\sum_{\ell^{\prime}, q^{\prime}, \mathbf{L}, \mathbf{q}} \frac{\mathbb{E}\left[\mathrm{e}^{\mathrm{j} 2 \pi\left(-\ell^{\prime T} \delta_{q^{\prime}}-\left(\ell^{\prime}-\ell_{1}\right)^{\mathrm{T}} \mathbf{x}_{q^{\prime}}-\left(\ell_{1}-\ell_{2}\right)^{\mathrm{T}} \mathbf{x}_{q_{1}}-\ldots-\left(\ell_{p}-\ell^{\prime}\right)^{\mathrm{T}} \mathbf{x}_{q_{p}}\right.}\right]}{(2 M+1)^{d} \boldsymbol{r}^{p}}
$$

equals $(26)$ since $f_{\delta}(z)=f_{\delta}(-z)$. Hence, $\phi\left(\mathbf{G} \widehat{\mathbf{G}}^{\dagger} \widehat{\mathbf{T}}^{p}\right)=\phi\left(\widehat{\mathbf{G}}^{\dagger} \mathbf{T}^{p}\right)$.

Equation (13) can be proved in a similar way using the procedure described above.

\section{PROOF OF (14) AND (15)}

From the definition (1), we have

$$
\begin{aligned}
& \underset{\Delta}{\mathbb{E}}\left[(\widehat{\mathbf{G}})_{\nu(\ell), q}\right]=\frac{\underset{\delta_{q}}{\mathbb{E}}\left[\mathrm{e}^{-\mathrm{j} 2 \pi \ell^{\mathrm{T}} \hat{\mathbf{x}}_{q}}\right]}{\sqrt{(2 M+1)^{d}}}=\frac{\mathrm{e}^{-\mathrm{j} 2 \pi \ell^{\mathrm{T}} \mathbf{x}_{q}} \underset{\delta_{q}}{\mathbb{E}}\left[\mathrm{e}^{-\mathrm{j} 2 \pi \ell^{\mathrm{T}} \delta_{q}}\right]}{\sqrt{(2 M+1)^{d}}} \\
& \mathrm{e}^{-\mathrm{j} 2 \pi \ell^{\mathrm{T}} \mathbf{x}_{q}} \prod_{m=1}^{d} \mathbb{E}\left[\mathrm{e}^{-\mathrm{j} 2 \pi \ell_{m} \delta_{m, q}}\right] \\
& =\frac{\delta_{\delta_{m, q}}}{\sqrt{(2 M+1)^{d}}}=(\mathbf{C})_{\nu(\ell), v(\ell)}(\mathbf{G})_{\nu(\ell), q},
\end{aligned}
$$

where $C_{\delta}(s)=\int_{-\infty}^{+\infty} \exp (s z) f_{\delta}(z) \mathrm{d} z$ is the characteristic function of $\delta$ and $\mathbf{C}$ is a diagonal matrix with entries given by $(\mathbf{C})_{\nu(\ell), v(\ell)}=\prod_{m=1}^{d} C_{\delta}\left(-\mathrm{j} 2 \pi \ell_{m}\right)$. We conclude that $\underset{\boldsymbol{\Delta}}{\mathbb{E}}[\widehat{\mathbf{G}}]=\mathbf{C G}$. Similarly the $\left(q, q^{\prime}\right)$-th element $\underset{\boldsymbol{\Delta}}{\mathbb{E}}\left[\widehat{\mathbf{G}}^{\dagger} \widehat{\mathbf{G}}\right]$, is given by

$$
\begin{aligned}
\underset{\Delta}{\mathbb{E}}\left[\left(\widehat{\mathbf{G}}^{\dagger} \widehat{\mathbf{G}}\right)_{q, q^{\prime}}\right] & =\frac{1}{(2 M+1)^{d}} \sum_{\ell} \underset{\Delta}{\mathbb{E}}\left[\mathrm{e}^{\mathrm{j} 2 \pi \ell^{\mathrm{T}} \hat{\mathbf{x}}_{q}} \mathrm{e}^{\left.-\mathrm{j} 2 \pi \ell^{\mathrm{T}} \hat{\mathbf{x}}_{q^{\prime}}\right]}\right. \\
& =\frac{1}{(2 M+1)^{d}} \sum_{\ell} \mathrm{e}^{\mathrm{j} 2 \pi \ell^{\mathrm{T}}\left(\mathbf{x}_{q}-\mathbf{x}_{q^{\prime}}\right)} \underset{\Delta}{\mathbb{E}}\left[\mathrm{e}^{\mathrm{j} 2 \pi \ell^{\mathrm{T}}\left(\delta_{q}-\delta_{q^{\prime}}\right)}\right]
\end{aligned}
$$

When $q \neq q^{\prime}$, we have

$$
\begin{aligned}
\underset{\Delta}{\mathbb{E}}\left[\left(\widehat{\mathbf{G}}^{\dagger} \widehat{\mathbf{G}}\right)_{q, q^{\prime}}\right] & =\frac{1}{(2 M+1)^{d}} \sum_{\ell} \mathrm{e}^{\mathrm{j} 2 \pi \ell^{\mathrm{T}}\left(\mathbf{x}_{q}-\mathbf{x}_{q^{\prime}}\right)} \underset{\delta_{q}}{\mathbb{E}}\left[\mathrm{e}^{\mathrm{j} 2 \pi \ell^{\mathrm{T}} \delta_{q}}\right] \underset{\delta_{q^{\prime}}}{\mathbb{E}}\left[\mathrm{e}^{-\mathrm{j} 2 \pi \ell \delta_{q^{\prime}}}\right] \\
& =\frac{1}{(2 M+1)^{d}} \sum_{\ell} \mathrm{e}^{\mathrm{j} 2 \pi \ell^{\mathrm{T}}\left(\mathbf{x}_{q}-\mathbf{x}_{q^{\prime}}\right)} \prod_{m=1}^{d} C_{\delta}\left(\mathrm{j} 2 \pi \ell_{m}\right) \prod_{m=1}^{d} C_{\delta}\left(-\mathrm{j} 2 \pi \ell_{m}\right) \\
& =\left(\mathbf{G}^{\dagger} \mathbf{C}^{\dagger} \mathbf{C G}\right)_{q, q^{\prime}} .
\end{aligned}
$$

Else, if $q=q^{\prime}$, we have:

$$
\underset{\Delta}{\mathbb{E}}\left[\left(\widehat{\mathbf{G}}^{\dagger} \widehat{\mathbf{G}}\right)_{q, q}\right]=\frac{1}{(2 M+1)^{d}} \sum_{\ell} \mathrm{e}^{\mathrm{j} 2 \pi \ell^{\mathrm{T}}\left(\hat{\mathbf{x}}_{q}-\hat{\mathbf{x}}_{q}\right)}=\frac{1}{(2 M+1)^{d}} \sum_{\ell} 1=1 .
$$

ACM Transactions on Sensor Networks, Vol. 6, No. 2, Article 11, Publication date: February 2010. 
Since $\mathbf{C}$ is diagonal and $\left(\mathbf{G}^{\dagger} \mathbf{C}^{\dagger} \mathbf{C G}\right)_{q, q}=\operatorname{Tr}\left\{\mathbf{C}^{\dagger} \mathbf{C}\right\} /(2 M+1)^{d}$, we conclude that $\underset{\Delta}{\mathbb{E}}\left[\widehat{\mathbf{G}}^{\dagger} \widehat{\mathbf{G}}\right]=\mathbf{G}^{\dagger} \mathbf{C}^{\dagger} \mathbf{C G}+\left(1-\frac{\operatorname{Tr}\left\{\mathbf{C}^{\dagger} \mathbf{C}\right\}}{(2 M+1)^{d}}\right) \mathbf{I}$.

D. DERIVATION OF (17) AND (19)

The result in (17) is obtained as follows. We apply to the first term in (16) the definition of $\phi(\cdot)$, the properties of the trace operator, and (15). Then, we have:

$$
\begin{aligned}
\beta^{2} \phi\left(\widehat{\mathbf{G}} \mathbf{G}^{\dagger}(\mathbf{T}+\alpha \beta \mathbf{I})^{-2} \mathbf{G} \widehat{\mathbf{G}}^{\dagger}\right) & =\beta^{2} \phi\left(\mathbf{G} \widehat{\mathbf{G}}^{\dagger} \widehat{\mathbf{G}} \mathbf{G}^{\dagger}(\mathbf{T}+\alpha \beta \mathbf{I})^{-2}\right) \\
& =\beta^{2} \phi\left(\mathbf{G} \underset{\Delta}{\mathbb{E}[}\left[\widehat{\mathbf{G}}^{\dagger} \widehat{\mathbf{G}}\right] \mathbf{G}^{\dagger}(\mathbf{T}+\alpha \beta \mathbf{I})^{-2}\right) \\
& =\phi\left(\mathbf{C}^{\dagger} \mathbf{C T}(\mathbf{T}+\alpha \beta \mathbf{I})^{-2} \mathbf{T}\right)+\beta \phi\left(\theta \mathbf{T}(\mathbf{T}+\alpha \beta \mathbf{I})^{-2}\right) .
\end{aligned}
$$

Similarly, using (15), the fourth term of (16) can be rewritten as:

$$
\begin{aligned}
\beta \Re \phi\left(\widehat{\mathbf{G}} \mathbf{G}^{\dagger}(\mathbf{T}+\alpha \beta \mathbf{I})^{-1}\right) & =\beta \Re \phi\left(\underset{\Delta}{\mathbb{E}}[\widehat{\mathbf{G}}] \mathbf{G}^{\dagger}(\mathbf{T}+\alpha \beta \mathbf{I})^{-1}\right) \\
& =\Re \phi\left(\mathbf{C} \mathbf{T}(\mathbf{T}+\alpha \beta \mathbf{I})^{-1}\right) .
\end{aligned}
$$

As for the result in (19), this is obtained as follows:

$$
\begin{aligned}
\phi\left(\mathbf{C}^{p}\right) & =\lim _{M, r \rightarrow+\infty} \frac{\operatorname{Tr}\left\{\mathbf{C}^{p}\right\}}{(2 M+1)^{d}}=\lim _{M, r \rightarrow+\infty} \frac{\sum_{\ell} \prod_{m=1}^{d} C_{\delta}\left(-\mathrm{j} 2 \pi \ell_{m}\right)^{p}}{(2 M+1)^{d}} \\
& =\lim _{M, r \rightarrow+\infty} \frac{\sum_{\ell} \prod_{m=1}^{d} \mathrm{e}^{-2 \pi^{2} p \sigma_{\delta}^{2} \ell_{m}^{2}}}{(2 M+1)^{d}}=\lim _{M, r \rightarrow+\infty} \frac{\sum_{\ell} \mathrm{e}^{-2 \pi^{2} p \rho^{2} \ell^{\mathrm{T}} \ell / r^{\prime 2}}}{(2 M+1)^{d}} \\
& =\lim _{M \rightarrow+\infty} \frac{\sum_{\ell} \mathrm{e}^{-2 \pi^{2} p \rho^{2} \beta^{\frac{2}{d}} \frac{\ell^{\mathrm{T} \ell}}{(2 M+1)^{2}}}}{(2 M+1)^{d}}=\int_{\mathcal{H}} \exp \left(-2 \pi^{2} p \rho^{2} \beta^{\frac{2}{d}} \mathbf{z}^{\mathrm{T}} \mathbf{z}\right) \mathrm{d} \mathbf{z} \\
& =\left(\frac{1}{\sqrt{2 \pi \beta^{\frac{2}{d}} \rho^{2} p}} \operatorname{erf}\left(\pi \beta^{\frac{1}{d}} \rho \sqrt{\frac{p}{2}}\right)\right)^{d} .
\end{aligned}
$$

\section{E. PROOF OF (20) AND (21)}

By using the definitions, we provided for $\phi(\cdot)$ and $\mathbf{T}$, we can write:

$$
\begin{aligned}
& \phi\left(\mathbf{T}^{2}\right)=\lim _{\substack{M, r \rightarrow+\infty \\
\beta}} \frac{1}{(2 M+1)^{d}} \mathbb{E}\left[\operatorname{Tr}\left\{\mathbf{T}_{\mathbf{x}}^{2}\right\}\right] \\
& =\lim _{M, r \rightarrow+\infty} \frac{1}{(2 M+1)^{d}} \mathbb{E}\left[\sum_{\ell_{1}}\left(\mathbf{T}_{\mathbf{x}}^{2}\right)_{v\left(\ell_{1}\right), v\left(\ell_{1}\right)}\right] \\
& =\lim _{M, r \rightarrow+\infty} \frac{1}{(2 M+1)^{d}} \mathbb{E}\left[\sum_{\ell_{1}, \ell_{2}}(\mathbf{T})_{v\left(\ell_{1}\right), v\left(\ell_{2}\right)}(\mathbf{T})_{v\left(\ell_{2}\right), v\left(\ell_{1}\right)}\right] \\
& =\lim _{M, r \rightarrow+\infty} \frac{1}{(2 M+1)^{d} r^{2}} \sum_{\ell_{1}, \ell_{2}} \sum_{q_{1}=1}^{r} \sum_{q_{2}=1}^{r} \mathbb{E}\left[\mathrm{e}^{-\mathrm{j} 2 \pi\left(\ell_{1}-\ell_{2}\right)^{\mathrm{T}} \mathbf{x}_{q_{1}}} \mathrm{e}^{-\mathrm{j} 2 \pi\left(\ell_{2}-\ell_{1}\right)^{\mathrm{T}} \mathbf{x}_{q_{2}}}\right] \\
& =L_{1}+L_{2} \text {, }
\end{aligned}
$$

ACM Transactions on Sensor Networks, Vol. 6, No. 2, Article 11, Publication date: February 2010. 
where $L_{1}$ is the contribution to $\phi\left(\mathbf{T}^{2}\right)$ when $q_{1}=q_{2}$, and $L_{2}$ is the contribution to $\phi\left(\mathbf{T}^{2}\right)$ when $q_{1} \neq q_{2}$. We have

$$
\begin{aligned}
L_{1} & =\lim _{M, r \rightarrow+\infty} \frac{1}{(2 M+1)^{d} r^{2}} \sum_{\ell_{1}, \ell_{2}} \sum_{q_{1}=1}^{r} \mathbb{E}\left[\mathrm{e}^{-\mathrm{j} 2 \pi\left(\ell_{1}-\ell_{2}\right)^{\mathrm{T}} \mathbf{x}_{q_{1}}-\mathrm{j} 2 \pi\left(\ell_{2}-\ell_{1}\right)^{\mathrm{T}} \mathbf{x}_{q_{1}}}\right] \\
& =\lim _{M, r \rightarrow+\infty} \frac{1}{(2 M+1)^{d} r^{2}} \sum_{\ell_{1}, \ell_{2}} \sum_{q_{1}=1}^{r} \mathbb{E}[1]=\lim _{M, r \rightarrow+\infty} \frac{(2 M+1)^{2 d} r}{(2 M+1)^{d} r^{2}}=\beta .
\end{aligned}
$$

As for $L_{2}$, we have

$$
L_{2}=\lim _{M, r \rightarrow+\infty} \sum_{\ell_{1}, \ell_{2}} \sum_{q_{1}=1}^{r} \sum_{\substack{q_{2}=1 \\ q_{2} \neq q_{1}}}^{r} \frac{\mathbb{E}\left[\mathrm{e}^{-\mathrm{j} 2 \pi\left(\ell_{1}-\ell_{2}\right)^{\mathrm{T}} \mathbf{x}_{q_{1}}}\right] \mathbb{E}\left[\mathrm{e}^{-\mathrm{j} 2 \pi\left(\ell_{2}-\ell_{1}\right)^{\mathrm{T}} \mathbf{x}_{q_{2}}}\right]}{(2 M+1)^{d} r^{2}}=L_{3}+L_{4}
$$

where $L_{3}$ is the contribution to $L_{2}$ when $\ell_{1}=\ell_{2}$ and $L_{4}$ is the contribution to $L_{2}$ when $\ell_{1} \neq \ell_{2}$. It turns out that:

$$
L_{3}=\lim _{M, r \rightarrow+\infty} \frac{1}{(2 M+1)^{d} r^{2}} \sum_{\ell_{1}} \sum_{q_{1}=1}^{r} \sum_{\substack{q_{2}=1 \\ q_{2} \neq q_{1}}}^{r} \mathbb{E}[1]=\lim _{\substack{M, r \rightarrow+\infty \\ \beta}} \frac{r(r-1)(2 M+1)^{d}}{(2 M+1)^{d} r^{2}}=1 .
$$

As for $L_{4}$, we assume that the random variables $x$ have average $\mathbb{E}[x]=\bar{x}$ and can be written as $x=\bar{x}+\tilde{x} / r^{\prime}$, with independent and identically distributed $\tilde{x}$. Hence,

$$
\begin{aligned}
\mathbb{E}\left[\mathrm{e}^{-\mathrm{j} 2 \pi \ell^{\mathrm{T}} \mathbf{x}}\right] & =\prod_{m=1}^{d} \mathbb{E}\left[\mathrm{e}^{-\mathrm{j} 2 \pi \ell_{m} x_{m}}\right]=\prod_{m=1}^{d} \int \mathrm{e}^{-\mathrm{j} 2 \pi \ell_{m} z} r^{\prime} f_{\tilde{x}}\left(r^{\prime} z-r^{\prime} \bar{x}_{m}\right) \mathrm{d} z \\
& =\prod_{m=1}^{d} \mathrm{e}^{-\mathrm{j} 2 \pi \ell_{m} \bar{x}_{m}} \int \mathrm{e}^{-\mathrm{j} 2 \pi \ell_{m} z^{\prime} / r^{\prime}} f_{\tilde{x}}\left(z^{\prime}\right) \mathrm{d} z^{\prime} \\
& =\mathrm{e}^{-\mathrm{j} 2 \pi \ell^{\mathrm{T}} \overline{\mathbf{x}}} \prod_{m=1}^{d} C_{\tilde{x}}\left(-\frac{\mathrm{j} 2 \pi \ell_{m}}{r^{\prime}}\right)=\mathrm{e}^{-\mathrm{j} 2 \pi \ell^{\mathrm{T}} \overline{\mathbf{x}}} \prod_{m=1}^{d} C_{\tilde{x}}\left(-\frac{\mathrm{j} 2 \pi \beta^{\frac{1}{d} \ell_{m}}}{2 M+1}\right) .
\end{aligned}
$$

ACM Transactions on Sensor Networks, Vol. 6, No. 2, Article 11, Publication date: February 2010. 
Note that we exploited the fact that: $f_{x}(z)=r^{\prime} f_{\tilde{x}}\left(r^{\prime} z-r^{\prime} \bar{x}\right)$. Thus, we can write:

$$
\begin{aligned}
& L_{4}=\lim _{M, r \rightarrow+\infty} \sum_{\ell_{1}} \sum_{\ell_{2} \neq \ell_{1}} \sum_{q_{1}=1}^{r} \sum_{\substack{q_{2}=1 \\
q_{2} \neq q_{1}}}^{r} \frac{\mathbb{E}\left[\mathrm{e}^{-\mathrm{j} 2 \pi\left(\ell_{1}-\ell_{2}\right)^{\mathrm{T}} \mathbf{x}_{q_{1}}}\right]}{(2 M+1)^{d} r^{2}} \mathbb{E}\left[\mathrm{e}^{-\mathrm{j} 2 \pi\left(\ell_{2}-\ell_{1}\right)^{\mathrm{T}} \mathbf{x}_{q_{2}}}\right] \\
& =\lim _{M, r \rightarrow+\infty} \frac{1}{(2 M+1)^{d} r^{2}} \sum_{\ell_{1}} \sum_{\ell_{2} \neq \ell_{1}} \sum_{q_{1}=1}^{r} \sum_{\substack{q_{2}=1 \\
q_{2} \neq q_{1}}}^{r} \prod_{m=1}^{d} C_{\tilde{x}}\left(-\mathrm{j} 2 \pi \beta^{\frac{1}{d}} \frac{\ell_{1, m}-\ell_{2, m}}{2 M+1}\right) \\
& \prod_{m=1}^{d} C_{\tilde{x}}\left(-\mathrm{j} 2 \pi \beta^{\frac{1}{d}} \frac{\ell_{2, m}-\ell_{1, m}}{2 M+1}\right) \mathrm{e}^{-\mathrm{j} 2 \pi\left(\ell_{1}-\ell_{2}\right)^{\mathrm{T}} \overline{\mathbf{x}}_{q_{1}}} \mathrm{e}^{-\mathrm{j} 2 \pi\left(\ell_{2}-\ell_{1}\right)^{\mathrm{T}} \overline{\mathbf{x}}_{q_{2}}} \\
& =\lim _{M, r \rightarrow+\infty} \frac{\beta}{(2 M+1)^{2 d}} \sum_{\ell_{1}} \sum_{\ell_{2} \neq \ell_{1}} \prod_{m=1}^{d}\left|C_{\tilde{x}}\left(-\mathrm{j} 2 \pi \beta^{\frac{1}{d}} \frac{\ell_{1, m}-\ell_{2, m}}{2 M+1}\right)\right|^{2} \\
& \cdot \sum_{q_{1}=1}^{r} \sum_{\substack{q_{2}=1 \\
q_{2} \neq q_{1}}}^{r} \mathrm{e}^{-\mathrm{j} 2 \pi\left(\ell_{1}-\ell_{2}\right)^{\mathrm{T}}\left(\overline{\mathbf{x}}_{q_{1}}-\overline{\mathbf{x}}_{q_{2}}\right)}
\end{aligned}
$$

since by definition $C\left(s^{*}\right)=C^{*}(s)$. Furthermore, if the averages $\overline{\mathbf{x}}_{q}$ are vertices of a $d$-dimensional regular grid in $\mathcal{H}$, we can replace the sums over the scalar indices $q_{1}$ and $q_{2}$ in (27), $q_{1}, q_{2}=1, \ldots, r$ with two $d$-dimensional sums over the vector indices $\mathbf{q}_{1}^{\prime}=\left[q_{11}^{\prime}, \ldots, q_{1 d}^{\prime}\right], q_{1 m}^{\prime}=1, \ldots, r^{\prime}$ and $\mathbf{q}_{2}^{\prime}=\left[q_{21}^{\prime}, \ldots, q_{2 d}^{\prime}\right]$, $q_{2 m}^{\prime}=1, \ldots, r^{\prime}$. Therefore,

$$
\sum_{q_{1}=1}^{r} \sum_{\substack{q_{2}=1 \\ q_{2} \neq q_{1}}}^{r} \mathrm{e}^{-\mathrm{j} 2 \pi\left(\ell_{1}-\ell_{2}\right)^{\mathrm{T}}\left(\overline{\mathbf{x}}_{q_{1}}-\overline{\mathbf{x}}_{q_{2}}\right)}=\sum_{\mathbf{q}_{1}^{\prime}} \sum_{\mathbf{q}_{2}^{\prime} \neq \mathbf{q}_{1}^{\prime}} \mathrm{e}^{-\mathrm{j} 2 \pi\left(\ell_{1}-\ell_{2}\right)^{\mathrm{T}}\left(\overline{\mathbf{x}}_{\mu\left(\mathbf{q}_{1}^{\prime}\right)}-\overline{\mathbf{x}}_{\mu\left(\mathbf{q}_{2}^{\prime}\right)}\right)}
$$

Notice that, if $\ell \neq \mathbf{0}$,

$$
\begin{aligned}
\sum_{\mathbf{q}^{\prime}} \mathrm{e}^{\left.-\mathrm{j} 2 \pi \ell^{\mathrm{T}} \overline{\mathbf{x}}_{\mu\left(\mathbf{q}^{\prime}\right)}\right)} & =\sum_{\mathbf{q}^{\prime}} \prod_{m=1}^{d} \mathrm{e}^{-\mathrm{j} 2 \pi \ell_{m} \bar{x}_{m, \mu\left(\mathbf{q}^{\prime}\right)}}=\prod_{m=1}^{d} \sum_{q_{m}^{\prime}=1}^{r^{\prime}} \mathrm{e}^{-\mathrm{j} 2 \pi \ell_{m} \bar{x}_{m, \mu}\left(\mathbf{q}^{\prime}\right)} \\
& =\prod_{m=1}^{d} \sum_{q_{m}^{\prime}=1}^{r^{\prime}} \mathrm{e}^{-\mathrm{j} 2 \pi \ell_{m}\left(\left(q_{m}^{\prime}-1 / 2\right) / r^{\prime}-1 / 2\right)}=0 .
\end{aligned}
$$

It follows that for $\ell_{1} \neq \ell_{2}$ (i.e., $\left.\left(\ell_{1}-\ell_{2}\right) \neq \mathbf{0}\right)$, we get

$$
\begin{aligned}
& \sum_{\substack{\mathbf{q}_{1}^{\prime} \\
\mathbf{q}_{2}^{\prime} \neq \mathbf{q}_{1}^{\prime}}} \mathrm{e}^{-\mathrm{j} 2 \pi\left(\ell_{1}-\ell_{2}\right)^{\mathrm{T}}\left(\overline{\mathbf{x}}_{\mu\left(\mathbf{q}_{1}^{\prime}\right)}-\overline{\mathbf{x}}_{\mu\left(\mathbf{q}_{2}^{\prime}\right)}\right)}=\sum_{\mathbf{q}_{1}^{\prime}} \mathrm{e}^{-\mathrm{j} 2 \pi\left(\ell_{1}-\ell_{2}\right)^{\mathrm{T}} \overline{\mathbf{x}}_{\mu\left(\mathbf{q}_{1}^{\prime}\right)}} \sum_{\mathbf{q}_{2}^{\prime} \neq \mathbf{q}_{1}^{\prime}} \mathrm{e}^{-\mathrm{j} 2 \pi\left(\ell_{2}-\ell_{1}\right)^{\mathrm{T}} \overline{\mathbf{x}}_{\mu\left(\mathbf{q}_{2}^{\prime}\right)}} \\
& =-\sum_{\mathbf{q}_{1}^{\prime}} \mathrm{e}^{-\mathrm{j} 2 \pi\left(\ell_{1}-\ell_{2}\right)^{\mathrm{T}} \overline{\mathbf{x}}_{\mu\left(\mathbf{q}_{1}^{\prime}\right)}} \mathrm{e}^{-\mathrm{j} 2 \pi\left(\ell_{2}-\ell_{1}\right)^{\mathrm{T}} \overline{\mathbf{x}}_{\mu\left(\mathbf{q}_{1}^{\prime}\right)}}=-\sum_{\mathbf{q}_{1}^{\prime}} 1=-r .
\end{aligned}
$$

ACM Transactions on Sensor Networks, Vol. 6, No. 2, Article 11, Publication date: February 2010. 
In conclusion,

$$
\begin{aligned}
L_{4} & =-\lim _{M, r \rightarrow+\infty} \frac{\beta}{(2 M+1)^{2 d}} \sum_{\ell_{1}} \sum_{\ell_{2} \neq \ell_{1}} \prod_{m=1}^{d}\left|C_{\tilde{x}}\left(-\mathrm{j} 2 \pi \beta^{\frac{1}{d}} \frac{\ell_{1, m}-\ell_{2, m}}{2 M+1}\right)\right|^{2} \\
& =-\beta\left(\int_{\mathcal{A}}\left|C_{\tilde{x}}\left(-\mathrm{j} 2 \pi \beta^{\frac{1}{d}}\left(z_{1}-z_{2}\right)\right)\right|^{2} \mathrm{~d} z_{1} \mathrm{~d} z_{2}\right)^{d},
\end{aligned}
$$

and $\phi\left(\mathbf{T}^{2}\right)=L_{1}+L_{3}+L_{4}=1+\beta-\beta\left(\int_{\mathcal{A}}\left|C_{\tilde{x}}\left(-\mathrm{j} 2 \pi \beta^{\frac{1}{d}}\left(z_{1}-z_{2}\right)\right)\right|^{2} \mathrm{~d} z_{1} \mathrm{~d} z_{2}\right)^{d}$ where $\mathcal{A}=\left\{\left(z_{1}, z_{2}\right) \in \mathbb{R}^{2} \mid z_{1} \in\left[-\frac{1}{2}, \frac{1}{2}\right), z_{2} \in\left[-\frac{1}{2}, \frac{1}{2}\right), z_{1} \neq z_{2}\right\}$. Also, for the position errors we have: $\hat{x}=\bar{x}+\frac{\tilde{x}+\tilde{\delta}}{r^{\prime}}$. By using the properties of the characteristic function, we can write: $C_{\tilde{x}+\tilde{\delta}}(s)=C_{\tilde{x}}(s) C_{\tilde{\delta}}(s)$ and

$$
\left.\phi\left(\widehat{\mathbf{T}}^{2}\right)=1+\beta-\beta\left(\int_{\mathcal{A}}\left|C_{\tilde{x}}\left(-\mathrm{j} 2 \pi \beta^{\frac{1}{d}}\left(z_{1}-z_{2}\right)\right)\right|^{2}\left|C_{\tilde{\delta}}\left(-\mathrm{j} 2 \pi \beta^{\frac{1}{d}}\left(z_{1}-z_{2}\right)\right)\right|^{2} \mathrm{~d} z_{1} \mathrm{~d} z_{2}\right)\right)^{d}
$$

\section{REFERENCES}

Akyildiz, I. F., Su, W., Sankarasubramaniam, Y., and Cayirci, E. 2002 . Wireless sensor networks: A survey. Comput. Netw. 38, 4, 393-422.

CRIstescu, R. AND Vetterli, M. 2005. On the optimal density for real-time data gathering of spatio-temporal processes in sensor networks. In Proceedings of the International Symposium on Information Processing in Sensor Networks (IPSN). ACM, New York, 159-164.

DAley, R. 1991. Atmospheric data analysis. Cambridge Atmospheric and Space Science Series.

DE Bruijn, N. 1958. Asymptotic Methods in Analysis. North-Holland, Amsterdam, The Netherlands.

Dogandžı'c, A. AND Zhang, B. 2005. Parametric signal estimation using sensor networks in the presence of node localization errors. In Proceedings of the 39th Asilomar Conference on Signals, Systems and Computers. IEEE Signal Processing Society, Los Alamitos, CA.

Dong, M., Tong, L., AND SADler, B. 2006. Impact of data retrieval pattern on homogeneous signal field reconstruction in dense sensor networks. IEEE Trans. Signal Processing 54, 11 (Nov.), 43524364.

EARLY, D. AND LONG, D. 2001. Image reconstruction and enhanced resolution imaging from irregular samples. IEEE Trans. Geosc. Remote Sens. 39, 2 (Feb.), 291-302.

Feichtinger, H. ANd GRöChenig, K. 1993. Error analysis in regular and irregular sampling theory. Appl. Anal. 50, 167-189.

Feichtinger, H. G., Gröchenig, K., AND Strohmer, T. 1995. Efficient numerical methods in nonuniform sampling theory. Numer. Math. 69, 423-440.

Ganesan, D., Ratnasamy, S., Wang, H., And Estrin, D. 2004. Coping with irregular spatio-temporal sampling in sensor networks. ACM SIGCOMM 34, 1 (Jan.), 125-130.

KAY, S. 1993. Fundamentals of statistical signal processing: Estimation theory. Prentice-Hall, Englewood Cliffs, NJ.

Kobayashi, M., Boutros, J., And Caire, G. 2001. Successive interference cancellation with siso decoding and em channel estimation. IEEE J. Select. Areas Commun. 19, 8 (Aug.).

Liv, B. And Stanley, T. 1965. Error bounds for jittered sampling. IEEE Trans. Autom. Contr. 10, 4, $449-454$.

LiU, J., Zhang, Y., AND ZhaO, F. 2006. Robust distributed node localization with error management. In Proceedigs of the 7th ACM International Symposium on Mobile Ad Hoc Networking and Computing (MobiHoc'06). ACM, New York, 250-261.

Marvasti, F. 2001. Nonuniform Sampling: Theory and Practice. Kluwer Academic: Plenum.

Marziliano, P. and Vetterli, M. 2000. Reconstruction of irregularly sampled discrete-time bandlimited signals with unknown sampling locations. IEEE Trans. Signal Proc. 48, 12 (Dec.), 34623471.

ACM Transactions on Sensor Networks, Vol. 6, No. 2, Article 11, Publication date: February 2010. 
Moore, D., Leonard, J., Rus, D., AND Teller, S. 2004. Robust distributed network localization with noisy range measurements. In Proceedings of the 2nd ACM Conference on Embedded Networked Sensor Systems (SenSys). ACM, New York, 50-61.

Nordio, A., Chiasserini, C.-F., AND Viterbo, E. 2006. Bandlimited field reconstruction for wireless sensor networks. arXiv:hep-th/0707.1954v1.

Nordio, A., Chiasserini, C.-F., And Viterbo, E. 2008. Performance of linear field reconstruction techniques with noise and uncertain sensor locations. IEEE Trans. Signal Proc. 56, 8 (Aug.), $3535-3547$.

Rachlin, Y., Negi, R., AND Khosla, P. 2005. Sensing capacity for discrete sensor network applications. In Proceedings of the International Symposium on Information Processing in Sensor Networks (IPSN). ACM, New York, 126-132.

RaUhuT, H. 2007. Random sampling of sparse trigonometric polynomials. Appl. Computat. Harmon. Anal. 22, 1, 16-42.

RAuth, M. ANd Strohmer, T. 1997. A frequency domain approach to the recovery of geophysical potentials. In Proceedings of the 1997 Workshop on Sampling Theory and Applications (Samp TA 97). 109-114.

Smale, S., AND Zhou, D.-X. 2004. Shannon sampling and function reconstruction from point values. Bull. Amer. Math. Soc. (N.S.) 41, 3, 279-305.

Stoica, P. and Moses, R. 2000. Introduction to Spectral Analysis. Prentice-Hall, Upper Saddle River, NJ.

Strohmer, T. 1993. Efficient methods for digital signal and image reconstruction from nonuniform samples. Ph.D. dissertation, University of Vienna, Vienna, Austria.

Strohmer, T., Binder, T., AND Süssner, M. 1996. How to recover smooth object boundaries in noisy medical images. In Proceedings of the IEEE International Conference on Image Processing (ICIP 96). IEEE Computer Society Press, Los Alamitos, CA, 331-334.

Sung, Y., Tong, L., And Poor, H. 2005. Sensor activation and scheduling for field detection in large sensor arrays. In Proceedings of the International Symposium on Information Processing in Sensor Networks (IPSN). ACM, New York.

TAYLOR, C., RAHIMI, A., AND BACHRACH, J. 2006. Simultaneous localization, calibration, and tracking in an ad hoc sensor network. In Proceedings of the International Symposium on Information Processing in Sensor Networks (IPSN). ACM, New York.

Tulino, A. And Verdú, S. 2004. Random matrix theory and wireless communications. Foundations and Trends in Communications and Information Theory. Now Publishers, Inc., Massachusetts.

VuRAN, M., AkAn, O., AND AkYILDIZ, I. 2004. Spatio-temporal correlation: Theory and applications for wireless sensor networks. Comput. Netw. 45, 3 (June), 245-259.

Weisstein, E. 2008. Euler-mascheroni constant. from MathWorld-A Wolfram Web Resource.

Zhao, P., Zhao, C., and Casazza, P. 2006. Perturbation of regular sampling in shift-invariant spaces for frames. IEEE Trans. Inf. Theory 52, 10 (Oct.), 4643-4648.

Received July 2007; revised February 2008, July 2008, February 2009, April 2009; accepted April 2009 